\title{
Ascaroside signaling in C. elegans ${ }^{*}$
}

\author{
Andreas H. Ludewig ${ }^{1}$, Frank C. Schroeder ${ }^{2 \S}$ \\ ${ }^{1}$ Institute of Human Nutrition and Food Science, Molecular Prevention, Christian Albrecht \\ University of Kiel, Heinrich-Hecht-Platz 10, 24118 Kiel, Germany \\ ${ }^{2}$ Boyce Thompson Institute and Department of Chemistry and Chemical Biology, Cornell \\ University, 1 Tower Road, Ithaca, NY 14853, USA
}

\section{Table of Contents}

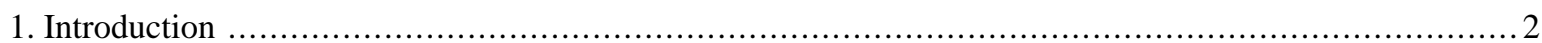

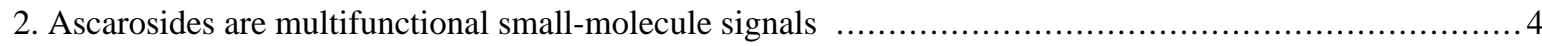

2.1. Ascarosides and dauer formation .............................................................. 4

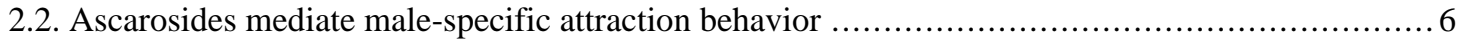

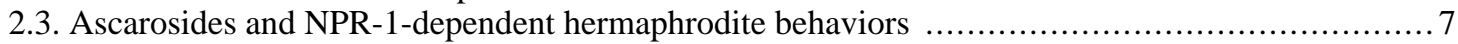

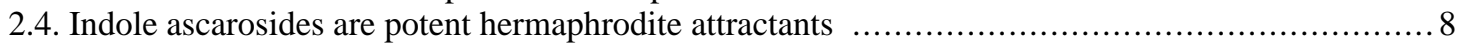

2.5. Male-produced ascarosides attract hermaphrodites ............................................. 10

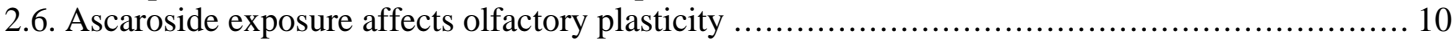

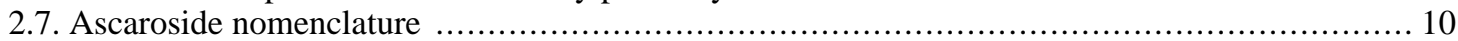

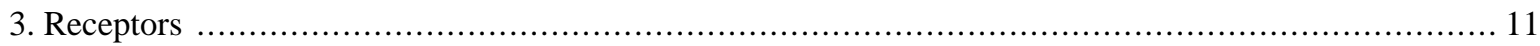

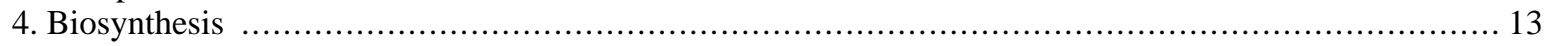

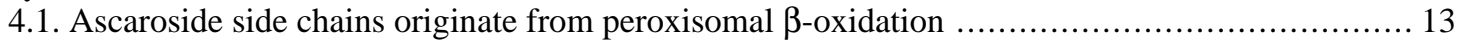

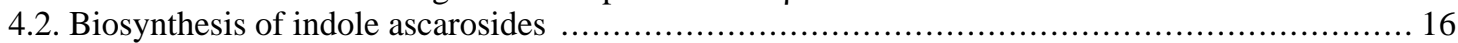

4.3. Ascarylose biosynthesis, side-chain functionalization, and a preliminary model ................... 16

4.4. Life stage and environmental conditions affect ascaroside production ............................. 16

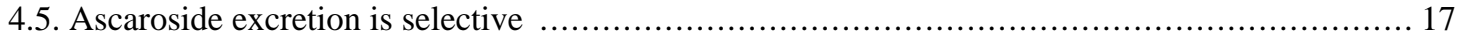

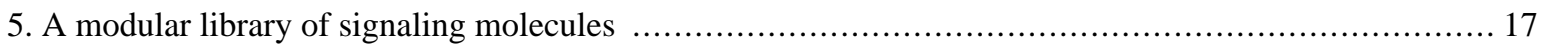

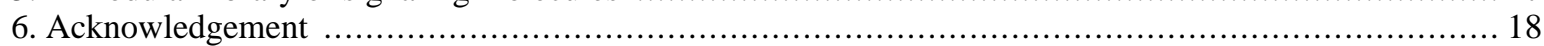

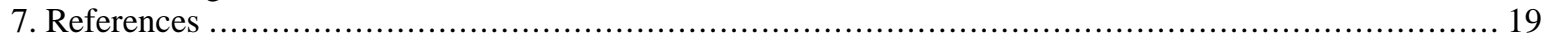

\footnotetext{
*Edited by Patty Kuwabara. Last revised October 15, 2012. Published January 18, 2013. This chapter should be cited as: Ludewig AH. and Schroeder FC. Ascaroside signaling in C. elegans (January 18, 2013), WormBook, ed. The C. elegans Research Community, WormBook, doi/10.1895/wormbook.1.155.1, http://www.wormbook.org.

Copyright: (c) 2012 Ludewig AH. and Schroeder FC. This is an open-access article distributed under the terms of the Creative Commons Attribution License, which permits unrestricted use, distribution, and reproduction in any medium, provided the original author and source are credited.

${ }^{\S}$ To whom correspondence should be addressed. E-mail: schroeder@ cornell.edu
} 


\begin{abstract}
Over the past 10 years, the relevance of small-molecule signaling for many aspects of C. elegans development and behavior has become apparent. One prominent group of small-molecule signals are the ascarosides, which control dauer entry and exit as well as a variety of sex-specific and social behaviors, including male attraction, hermaphrodite repulsion, olfactory plasticity, and aggregation. This wide range of biological functions is facilitated by a great diversity of ascaroside chemical structures. These are based on the sugar ascarylose, which is linked to fatty acid-like side chains of varying lengths and often decorated further with building blocks derived from amino acids, folate, and other primary metabolites. Different ascarosides or combinations of ascarosides mediate different phenotypes, and even small differences in chemical structures are often associated with strongly altered activity profiles. Additional complexity arises from concentration-dependent effects and synergism between different ascarosides. The ascarosides are sensed by several types of chemosensory head neurons, including the ASK, ASI, and ADL neurons as well as the male-specific CEM neurons. Ascaroside perception is mediated by diverse families of G-protein coupled membrane receptors that act upstream of conserved signal transduction pathways, including insulin/IGF-1 signaling and transforming growth factor beta (TGF- $\beta$ ) signaling. Biosynthesis of the ascarosides appears to integrate input from several primary metabolic pathways, including peroxisomal $\beta$-oxidation of long-chain fatty acids and amino acid catabolism. Life stage, sex, as well as food availability and other environmental factors affect ascaroside biosynthesis, suggesting that ascaroside signaling communicates detailed information about life history and metabolic state.
\end{abstract}

\title{
1. Introduction
}

The term "ascarosides" was introduced originally to refer to a distinct type of lipid first detected in parasitic roundworms of the family Ascaridia more than 100 years ago. In 1912, Flury reported a remarkably detailed chemical analysis of the human intestinal parasite Ascaris lumbricoides, which included isolation of an "unsaponifiable matter" of then unknown composition that accounted for about $25 \%$ of the total lipid content of this nematode (Flury, 1912). Flury commented on the high fat content of the worms he analyzed as follows:

The high fat content of the dried animals reveals itself by the fact that, once ignited, they continue to burn like a candle, with a brightly luminous flame (Flury 1912). ${ }^{1}$

A first attempt at determining the chemical structures of the Ascaris-derived ascarosides by Schulz and Becker in 1933 suggested a molecular formula of $\mathrm{C}_{33} \mathrm{H}_{68} \mathrm{O}_{4}$ (Schulz and Becker, 1933), but it was not until 1957 that the exact structures of the ascarosides found in Ascaris spp. were elucidated (Fairbairn, 1957; Fouquey et al., 1957). Chemically, ascarosides are defined as glycosides of the dideoxysugar ascarylose (Figure 1). The ascarosides obtained from Ascaris feature very long aliphatic side chains (predominantly 29 and more carbons in length). These very lipophilic ascarosides were shown to form a protective coating of Ascaris eggs, providing remarkable resilience to harsh environmental conditions and high concentrations of toxic or noxious chemicals (Fairbairn, 1957; Foor, 1967; Jezyk and Fairbairn, 1967). More recently, additional variants of such lipophilic ascarosides have been described from Ascaris suum (Bartley et al., 1996).

\footnotetext{
${ }^{1}$ Translated from German: "Der relativ hohe Fettgehalt der getrockneten Tiere zeigt sich auch darin, daß sie beim Anzünden wie eine Kerze mit leuchtender Flamme weiter brennen."
} 


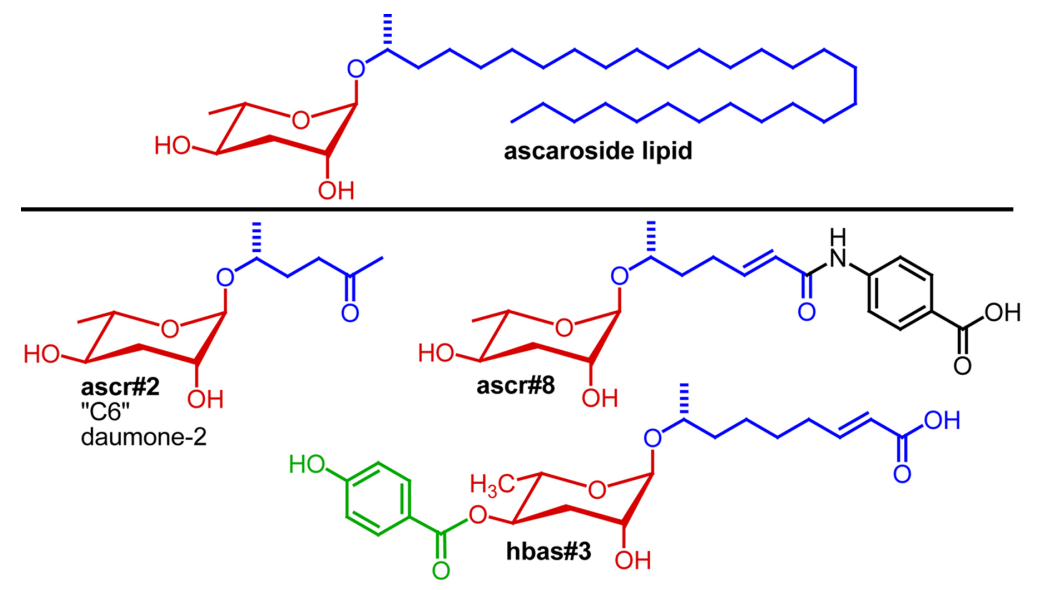

Figure 1. Lipophilic ascaroside from Ascaris (top) and examples for small molecule signals based on ascarosides from C. elegans.

Over the past seven years, a large number of more hydrophilic and structurally much more varied ascaroside derivates has been identified from C. elegans and other nematodes. These hydrophilic ascarosides are important small-molecule signals that regulate a remarkable variety of biological processes in nematodes, including developmental timing, reproduction, and social signaling. The structures, biosyntheses, and biological roles of this functionally diverse family of signaling molecules form the subject of this chapter.

In C. elegans, ascarosides were first identified as signaling molecules regulating the population density-dependent formation of dauer larvae. C. elegans larvae interrupt normal reproductive development and enter the dauer diapause in response to adverse environmental conditions and high population density (recently reviewed in $\mathrm{Hu}, 2007)$. Dauer larvae do not feed and are highly stress resistant and long lived (up to 8 months). Upon improvement of environmental conditions, dauer larvae resume normal development resulting in reproductive adults with a normal lifespan (Cassada and Russell, 1975; Klass and Hirsh, 1976). Extensive mutant screens led to identification of about 40 dauer formation defective (Daf) mutants that are either unable to form dauers (dauer formation defective, Daf-d) or constitutively form dauer larvae (Daf-c) (Albert and Riddle, 1988; Riddle et al., 1981). Epistasis analysis of $d a f$ genes revealed a complex signaling network that regulates dauer formation and exit and is based on conserved signaling pathways including steroid hormone, serotonin, cGMP, TGF- $\beta$, and insulin/IGF signaling.

In 1982, Golden and Riddle demonstrated that metabolite extracts of C. elegans cultures induce dauer formation and antagonize recovery from dauer, presumably due to the presence of a small-molecule signal produced by the worms (Golden and Riddle, 1982). The identification of a mutant strain, daf-22, that did not produce dauer-inducing activity, confirmed that this signal must be worm-derived and further demonstrated that it is required for dauer entry (Golden and Riddle, 1985). Based on solubility and other physical properties of the dauer-inducing signal, Golden and Riddle suggested that this "dauer pheromone" may consist of a mixture of short-chain hydroxylated lipid derivatives (Golden and Riddle, 1982; Golden and Riddle, 1984). The eventual identification of ascarosides as dauer pheromone constituents vindicated this hypothesis; however, the exact chemical nature of the dauer pheromone remained unknown for over two decades after its initial discovery. Despite the lack of such detailed chemical knowledge, dauer pheromone and its effects on insulin/IGF, TGF- $\beta$, and other conserved signaling pathways were studied extensively (Larsen et al., 1995).

More than 20 years after the initial discovery, the chemical composition of the dauer pheromone was finally revealed. Several laboratories contributed to identification of a family of hydrophilic ascarosides with short three- to nine -carbon side chains as the major components of the dauer pheromone (Butcher et al., 2007; Jeong et al., 2005; Pungaliya et al., 2009). It became apparent that the dauer pheromone does not consist of just one or two compounds, but instead represents a complex mixture of ascarosides, in which some components act synergistically in dauer induction (Section 2.1).

Following their identification as constituents of the dauer pheromone, it was discovered that ascarosides serve additional important signaling functions. Srinivasan et al. found that distinct concentrations and combinations of ascarosides are attractive to male C. elegans, but repulsive to hermaphrodites (Section 2.2) (Srinivasan et al., 2008). Subsequently, Macosko et al. uncovered the neuronal network regulating hermaphrodite repulsion by ascarosides 
(Section 2.3) (Macosko et al., 2009). Most recently, it was shown that indole ascarosides and p-hydroxybenzoyl ascarosides act as highly potent aggregation signals that attract both males and hermaphrodites (Section 2.4) (Srinivasan et al., 2012; von Reuss et al., 2012), whereas primarily male-produced ascarosides attract specifically hermaphrodites (Section 2.5) (Izrayelit et al., 2012). Furthermore, ascarosides have been shown to affect olfactory plasticity (Section 2.6) (Yamada et al., 2010). Ascaroside receptors, biosynthesis, and chemical analysis are discussed in Sections 3 and 4 of this chapter.

\section{Ascarosides are multifunctional small-molecule signals}

\subsection{Ascarosides and dauer formation}

For more than 20 years after the initial description of the dauer pheromone as a small molecule signal by Golden and Riddle (Golden and Riddle, 1982), its chemical identity remained enigmatic. Finally, in 2005 Jeong et al. described the isolation and identification of "daumone" (also "ascr\#1", or "C7": see Section 2.7. for ascaroside nomenclature), an ascaroside featuring a seven-carbon carboxylic acid side chain, as one component of the dauer pheromone (Jeong et al., 2005). Isolation of ascr\#1 was based on activity-guided fractionation of $C$. elegans liquid culture extracts, using dauer formation for tracking activity. However, because of its relatively low specific activity, ascr\#1 could not account for most of the dauer-inducing activity of $C$. elegans liquid culture extracts ("natural dauer pheromone") (Butcher et al., 2007; Gallo and Riddle, 2009; Jeong et al., 2005). For example, Gallo and Riddle compared the physiological properties of ascr\#1 with those of natural dauer pheromone and found that at the concentrations required to induce dauer ascr\#1 has significant toxicity (Gallo and Riddle, 2009). These observations suggested that additional dauer pheromone components must exist, which was verified by a series of subsequent studies that showed that the dauer pheromone consists of a highly complex mixture of structurally varied ascarosides. Using activity-guided fractionation approaches, Clardy and co-workers identified two additional ascarosides with stronger dauer-inducing activity, featuring a six-carbon side chain bearing a ketone functionality ("C6", ascr\#2) and a nine-carbon $\alpha, \beta$-unsaturated carboxylic acid side chain ("C9", ascr\#3) (Butcher et al., 2007) (Figure 2). A fourth ascaroside, bearing a three-carbon side chain ("C3" or ascr\#5) was found to synergize with ascr\#2 and ascr\#3 in dauer formation (Butcher et al., 2008) (Figure 2). The synergistic activity of dauer pheromone components suggested that ascaroside perception may involve several different receptors with different affinities for different ascarosides. Furthermore, it was shown that dauer pheromone composition depends on cultivation conditions: dauer pheromone extract derived from liquid cultures grown at $20^{\circ} \mathrm{C}$ contained much less ascr\#1 than extracts from cultures grown at $25^{\circ} \mathrm{C}$, indicating that environmental conditions influence the molecular composition of the dauer pheromone cocktail (Butcher et al., 2007; Jeong et al., 2005; Lee et al., 2010).

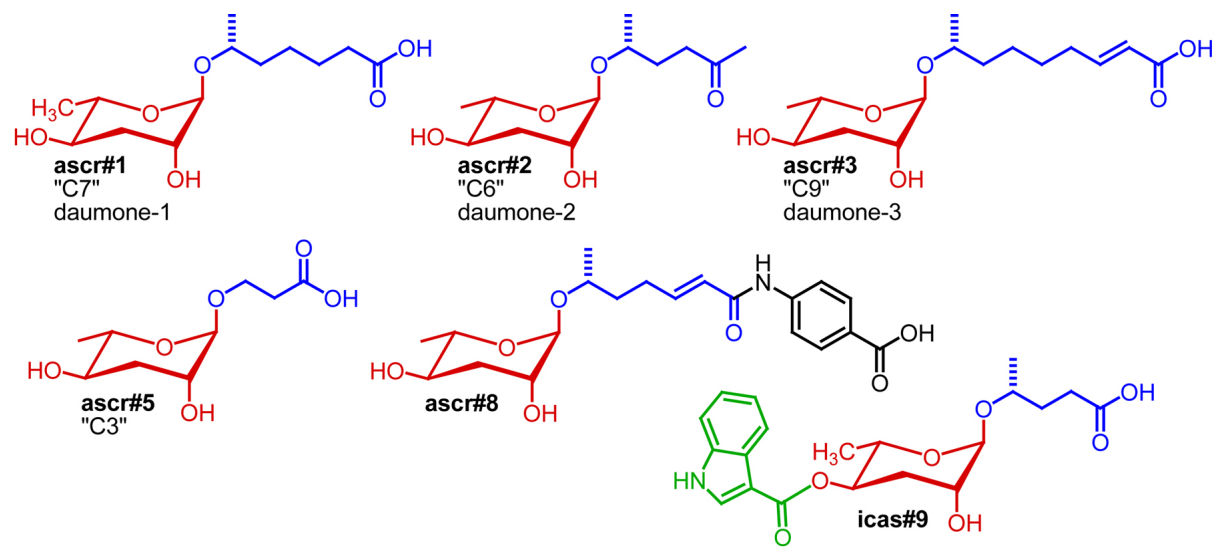

Figure 2. Ascarosides that have been shown to induce dauer formation.

The finding that the dauer pheromone consists of a synergistic mixture explained the difficulties experienced with identification of the chemical nature of the pheromone via activity-guided fractionation: separation of different components that synergize must lead to partial loss of activity, greatly complicating the task of attributing activity to individual compounds. Therefore, Pungaliya et al. explored applicability of NMR spectroscopy-based comparative metabolomics to complete identification of the dauer pheromone's constituents (Pungaliya et al., 2009). These studies revealed several additional ascarosides, including ascr\#8, a potent component of the dauer pheromone, whose chemical structure differs significantly from those of the previously identified ascarosides. Ascr\#8 includes a 
$p$-aminobenzoic acid moiety, forming an amide with a seven-carbon $\alpha, \beta$-unsaturated fatty acid similar to the side chains found in ascr\#1 or ascr\#3 (Figure 2). p-Aminobenzoic acid is uncommon among known primary and secondary metabolites; except for it forming an integral part of folate. Additional ascarosides identified via NMR-based comparative metabolomics include ascr\#7, representing an $\alpha, \beta$-unsaturated analog of ascr\#1 (daumone), and the two diastereomeric alcohols ascr\#6.1 and ascr\#6.2; however, none of these compounds was shown to confer significant additional dauer-inducing activity. In a parallel effort, Butcher et al. identified another intriguing structural variant, the indole ascaroside icas\#9 (also "C5" or "indole ascaroside C5") featuring an unusual indole carboxy moiety attached to the ascarylose sugar (Figure 2). The activity profile of icas\#9 differs from that of other ascarosides in that its activity declines at higher concentrations, possibly suggesting a different mode of receptor activation (Butcher et al., 2009).

Two recent studies employing NMR spectroscopy and mass spectrometry based comparative metabolomics suggest that the number of dauer pheromone components may be even larger. Srinivasan et al. showed that $C$. elegans produce an entire series of indole ascarosides, among which icas\#3 and the earlier described icas\#9 are the most abundant (Srinivasan et al., 2012). These indole ascarosides were shown to function as potent aggregation pheromones (see Section 2.4), and it seems likely that they also modulate dauer formation. Subsequently, von Reuss et al. used mass spectrometry-based metabolomics to investigate ascaroside biosynthesis (see Section 4.1) which led to identification of a large number of additional ascarosides, including several structural derivatives of the strongly dauer inducing ascr\#3 (von Reuss et al., 2012). Examples include the saturated derivatives ascr\#10 and oscr\#10, the latter of which features a side chain that bears the ascarylose at the ultimate carbon ( $\omega$-functionalization) instead of the usual penultimate carbon ( $\omega$-1-functionalization) as in ascr\#10 (von Reuss et al., 2012) (Figure 3). This study also revealed a larger number of indole ascarosides as well as ascarosides featuring a $p$-hydroxybenzoyl- and (E)-2-methyl-2-butenoyl side chains (Figures 1 and 3). Some of these compounds have been shown to strongly affect worm behavior (von Reuss et al., 2012). Whether these additional ascarosides contribute significantly to dauer formation, inhibit dauer recovery, or synergize with other components of the dauer pheromone has not been investigated.

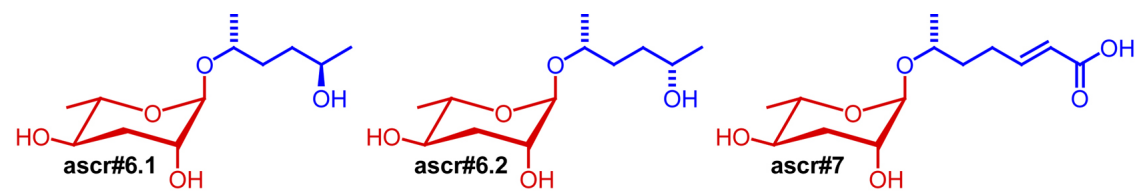

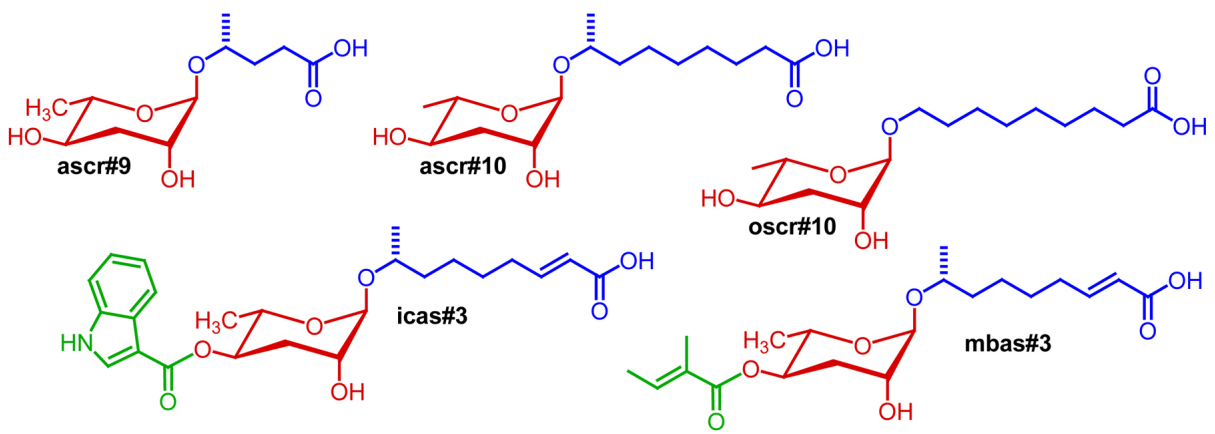

Figure 3. Ascarosides structurally related to the dauer-inducing compounds in Figure 2. A role of these ascarosides in dauer formation has not been demonstrated.

Using natural dauer pheromone, it has been demonstrated that the dauer pheromone signal is detected and integrated by specific chemosensory head neurons, including ADF, ASG, ASI, ASJ, and, to a lesser extent, ASK neurons (Bargmann, 2006). When exposed to pheromone, these neurons block or in a few cases initiate the production of secondary messengers including TGF-B, cGMP, serotonin and insulin-like peptides (Figure 4). These neuroendocrine pathways transduce the dauer signal to elicit systemic responses in various tissues (Riddle and Albert, 1997). Most of these experiments have not yet been repeated with synthetic ascarosides or ascaroside mixtures in place of natural pheromone. In one study, Kim et al. showed that synthetic ascarosides repress $d a f-7$ TGF- 3 expression similar to natural dauer pheromone, with ascr\#2, ascr\#3, and ascr\#5 being more potent than ascr\#1, confirming that different ascarosides have different potencies (Kim et al., 2009). However, it remains largely unknown whether different ascarosides are associated with different pathways (and neurons), or instead all ascarosides signal via the same pathways. 


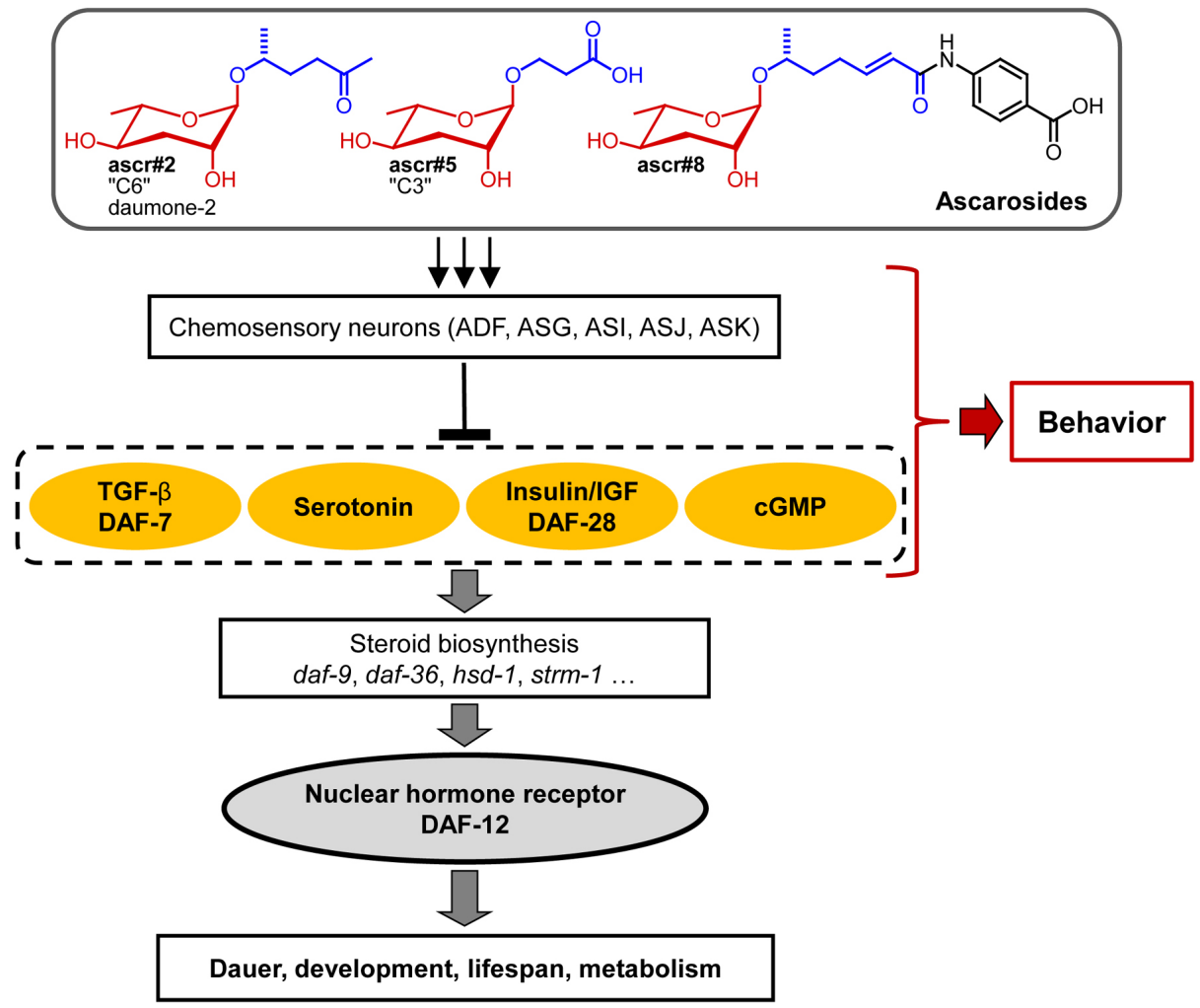

Figure 4. Simplified signaling network regulating development, lifespan, and metabolism in $C$. elegans, highlighting the roles of small-molecule signals. Ascarosides function upstream of several conserved signaling pathways that control the synthesis or availability of steroid hormones, the dafachronic acids, which serve as ligands of the nuclear hormone receptor DAF-12, a central regulator of $C$. elegans life history and a homolog of mammalian vitamin $D$ and liver $X$ receptors (Fielenbach and Antebi, 2008). The role of ascarosides in modulating behavior is discussed in Sections 2.2.-2.6.

The dauer pheromone signal finally converges on the cytochrome P450 DAF-9 and on the nuclear hormone receptor DAF-12 (Antebi et al., 2000; Gerisch et al., 2001; Lee and Schroeder, 2012). Under dauer inducing conditions, DAF-9 expression is strongly reduced or abolished and the steroidal ligands of DAF-12, the dafachronic acids, are not produced. As a result, the unliganded DAF-12 recruits its co-factor DIN-1 and triggers expression of dauer specific gene (Figure 4) (Bethke et al., 2009; Ludewig et al., 2004; Motola et al., 2006). Accordingly, exposure to natural dauer pheromone downregulates DAF-9 expression and thus dafachronic acid biosynthesis; however, this has not yet been demonstrated for synthetic ascaroside blends (Schaedel et al. 2012). Current knowledge of ascaroside perception and their receptors is summarized in Section 3.

\subsection{Ascarosides mediate male-specific attraction behavior}

C. elegans males display a broad spectrum of sex-specific behaviors that relate to mating (Barr and Garcia, 2006; Chasnov and Chow, 2002; Chasnov et al., 2007). In 2002, Simon and Sternberg showed that hermaphrodites produce a chemical cue, likely a small molecule signal, that attracts males (Simon and Sternberg, 2002). The existence of a male-attracting pheromone was further confirmed by studies of White et al., who showed that males are attracted to metabolite extracts from hermaphrodite liquid cultures (White et al., 2007). Laser-ablation studies showed that perception of the attractive signal required the male-specific cephalic companion (CEM) neurons, along with the AWA and AWC olfactory neurons. White et al. further showed that male response to hermaphrodite pheromone is mediated by a TRPV (transient receptor potential vanilloid) channel dependent on osm-9, ocr-1, and ocr-2. (White et al., 2007). Chasnov et al. compared male attraction of several different Caenorhabditis species and showed that $C$. elegans males are more strongly attracted to females of other Caenorhabditis species than to $C$. elegans hermaphrodites, suggesting that $C$. elegans hermaphrodites may have lost the ability to produce some male-attracting compounds, or produce these in smaller amounts than females from other Caenorhabditis species (Chasnov et al., 2007).

For chemical characterization of the male attracting signal, Srinivasan et al. pursued activity-guided fractionation of $C$. elegans metabolite extracts (Srinivasan et al., 2008), but in contrast to identification of the dauer 
pheromone, special measures were taken to avoid contamination with bacterial small molecules. This greatly simplified the chemotaxis assays as worms are attracted to many bacterial small molecules. Using synchronized batches of worms, the time window during which the male-attracting substance(s) were produced was determined. It was found that L4, young adult, and adult hermaphrodites produce the male-attracting factor, with the greatest amounts in L4 larvae and young adults (Srinivasan et al., 2008). Chemical analyses revealed that male-attracting activity is due to two major dauer pheromone constituents, ascr\#2 and ascr\#3. These two ascarosides strongly attract males at concentrations far below those required for dauer induction. The activity of ascr\#2 and ascr\#3 is further enhanced by another ascaroside, ascr\#4, which chemically represents a glucosylated ascr\#2 (Figure 5). Mixtures of ascr\#2, ascr\#3, and ascr\#4 are much more potently attractive than the individual components, confirming that these compounds act in synergy. In contrast to their activity profiles in dauer formation, male-attracting activity of ascr\#2 and ascr\#3 decreases at higher concentrations, resulting in a bell-shaped activity profile. The bimodal activity profiles obtained for ascr\#2 and ascr\#3 in the male attraction assay suggest receptor desensitization at high compound concentrations, whereas the saturated dose-response curves obtained for ascr\#2 and ascr\#3 in the dauer formation assays suggests that that receptor desensitization may not be involved in dauer formation.

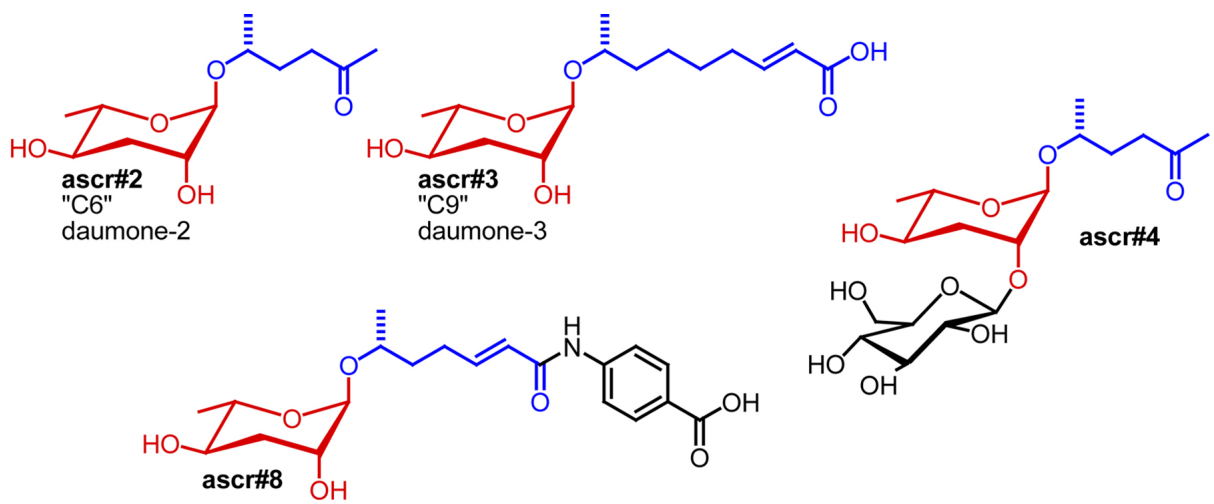

Figure 5. Structures of ascarosides that synergize in male attraction.

C. elegans males, but not hermaphrodites are attracted to mixtures of ascr\#2 and ascr\#3 at picomolar to low nanomolar concentrations. At higher, dauer inducing concentrations, males are no longer attracted whereas wild-type hermaphrodites show strong repulsion, indicating yet another function of ascarosides in social signaling (Srinivasan et al., 2008). The role of ascr\#2, ascr\#3, ascr\#5 and other ascarosides for hermaphrodite avoidance and aggregation behavior was further investigated as described in the following section (Macosko et al., 2009; Srinivasan et al., 2012).

As activity-guided fractionation had revealed that the male-attracting signal consists of a synergistic blend of ascarosides, it seemed possible that additional components may have been missed. Testing a series of additional ascarosides identified via NMR-based comparative metabolomics revealed that the dauer-inducing ascr\#8 synergizes with ascr\#2 and ascr\#3, strongly enhancing male attraction (Pungaliya et al., 2009). In contrast, several other ascarosides were found to be completely inactive in the male-attraction assay. For example, ascr\#1 and ascr\#7 are both inactive, although the chemically closely related ascr\#3, alongside ascr\#8, constitutes one of the two strongest male attractants (Pungaliya et al., 2009). These structure-activity relationships further suggested that ascaroside perception involves multiple, structure-specific receptors.

Male response to ascr\#3 was shown to require both the sex-specific CEM neurons and the amphid single-ciliated sensory neuron type K (ASK) (Srinivasan et al., 2008). Furthermore, ascr\#3 dependent male attraction was abolished in the general sensory mutants $o s m-6$ and $o s m-3$, which are expressed in ciliated neurons and are also required for dauer pheromone perception (Vowels and Thomas, 1992). In summary, these studies demonstrate that ascaroside signaling regulates both dauer formation and mating behavior in a concentration dependent manner, thus connecting reproductive and developmental pathways.

\subsection{Ascarosides and NPR-1-dependent hermaphrodite behaviors}

C. elegans exhibits natural variation in its foraging behavior. Some strains (e.g., the laboratory strain N2) disperse individually on a bacterial lawn and show a generally low tendency to aggregate, whereas most wild-type 
strains (for example, the Hawaii strain CB4856) aggregate where bacteria are most abundant (de Bono and Bargmann, 1998; Hodgkin and Doniach, 1997). These behavioral variants are generally referred to as "solitary", and "social,', respectively (de Bono and Bargmann, 1998; Rogers et al., 2003). These differences in aggregation behavior are associated with two different alleles of the neuropeptide Y receptor homolog NPR-1 that differ only at a single amino acid position: solitary strains such as N2 express a high-activity variant of NPR-1 (215-valine), whereas aggregating strains such as CB4856 express a low-activity variant of NPR-1 (215-phenylalanine). The strong loss-of-function mutants npr-1(ad609) and npr-1(ky13), which were generated in the N2 background, also show a high tendency to aggregate (de Bono and Bargmann, 1998; Hodgkin and Doniach, 1997). The interneuron RMG, the central site of action of NPR-1, is proposed to serve as a central hub computing aggregation and attraction signals originating from several different sensory neurons (Macosko et al., 2009), including the ASK neurons, which had been shown to be required for male attraction to ascarosides (Srinivasan et al., 2008).

Macosko et al. showed that loss of function of $n p r-1$ strongly affects hermaphrodite response to ascarosides (Macosko et al., 2009). Whereas NPR-1 high activity strains such as N2 are strongly repulsed by ascr\#2, ascr\#3, and ascr\#5 (Figure 6), mixtures of these compounds become attractive in npr-1 loss-of-function mutants. These npr-1 dependent effects of ascarosides are mediated by signaling from the ASK sensory neurons to the RMG interneuron where NPR-1 is expressed (Macosko et al., 2009). Thus ASK sensory neurons mediate attraction of males to ascr\#2 and ascr\#3 as well as attraction of $n p r-1$ loss-of-function hermaphrodites to ascr\#2, ascr\#3, and ascr\#5. However, it remained unclear how social wild-type hermaphrodites, carrying a low-activity variant of NPR-1 (215-phenylalanine), would respond to these ascarosides. A subsequent study showed that, in contrast to $n p r-1$ loss-of-function mutants, social wild-type hermaphrodites are either not attracted or show weak repulsion to mixtures of ascr\#2, ascr\#3, and ascr\#5 (Srinivasan et al., 2012).
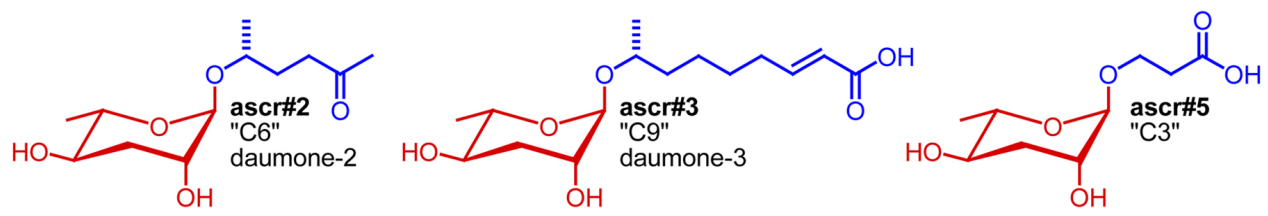

Figure 6. Mixtures of ascr\#2, ascr\#3, and ascr\#5 are repulsive to solitary and social wild-type hermaphrodites.

The starkly different responses of males and hermaphrodites to ascr\#3 (and perhaps other ascarosides) appear to result in part from antagonism of several different synaptic pathways, involving both the ADL and ASK sensory neurons (Jang et al., 2012). Additionally, hermaphrodite repulsion by ascarosides may result from sex-specific changes in the ASI neurons during development, which have been shown to result in repression of male-specific attraction behavior in adult hermaphrodites (White and Jorgensen, 2012), although the role of ascarosides in the observed attraction behaviors was not studied.

\subsection{Indole ascarosides are potent hermaphrodite attractants}

The indole ascaroside icas\#9 ("indolecarboxy ascaroside C5") was first discovered via activity-guided fractionation of dauer inducing metabolite fractions (Butcher et al., 2009) (see Section 2.1). Subsequent comparative metabolomic analyses of wild-type and $d a f-22$ mutant metabolomes revealed an entire series of ascarosides carrying an indolecarboxy moiety attached to position 4 of the ascarylose (Srinivasan et al., 2012). Indole ascarosides are much less abundant than the earlier discovered non-indole ascarosides (von Reuss et al., 2012). Notably, the indole ascarosides' quantitative distribution is distinctly different from that of the corresponding non-indole ascarosides, suggesting that incorporation of the indole unit is strongly regulated (Srinivasan et al., 2012). Stable isotope incorporation experiments demonstrated that the indole carboxy moiety is derived from the amino acid tryptophan (Srinivasan et al., 2012).

Behavioral assays showed that at high concentrations, the indole ascarosides icas\#3 and icas\#9 attract both hermaphrodites and males; whereas at lower, physiological concentrations only hermaphrodites are attracted (Srinivasan et al., 2012). Significant hermaphrodite attraction was observed at concentrations as low as $100 \mathrm{fM}$ (femtomolar). In contrast to non-indole ascarosides, icas\#3 is strongly attractive to hermaphrodites from all tested social and solitary strains, including $n p r-1$ loss-of-function mutants. In addition, icas\#3 increases hermaphrodite aggregation on food at concentrations as low as $10 \mathrm{fM}$ (Srinivasan et al., 2012). 
These results showed that specific structural variants of ascarosides are associated with specific functions, as addition of an indole carboxy group to repulsive ascr\#3 changes the signaling properties such that the indole-modified version icas\#3 functions as a strong attractant (Srinivasan et al., 2012) (Figure 7). Srinivasan et al. further showed that ascr\#3 and icas\#3 are competing signals. Physiological mixtures of ascr\#3 and icas\#3 are attractive at low total compound concentrations but repulsive at higher concentrations (Srinivasan et al., 2012).

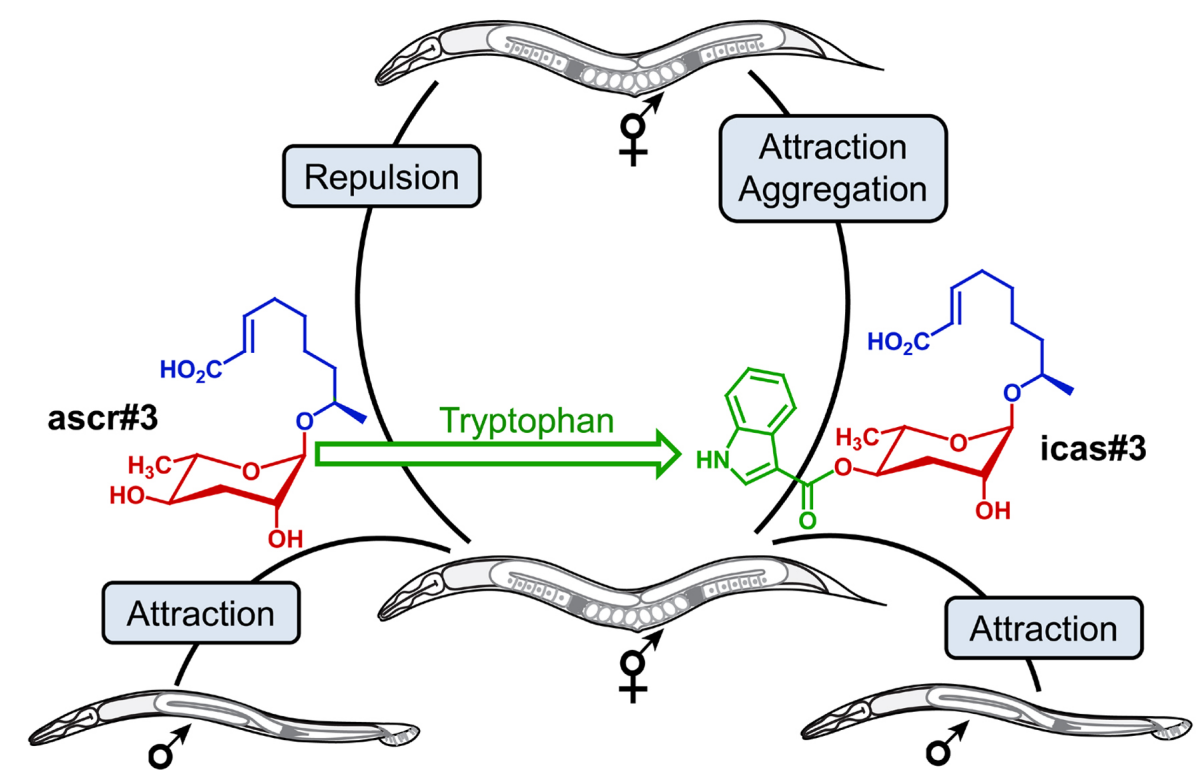

Figure 7. The non-indole ascaroside ascr\#3 (green) attracts males but is repulsive to hermaphrodites. Addition of the tryptophan-derived indole carboxy moiety (red) to repulsive ascr\#3 produces icas\#3, a potent hermaphrodite attractant (Srinivasan et al., 2012).

Attraction of hermaphrodites by icas\#3 requires the ASK sensory neurons, which are also involved in responses to the generally repulsive ascr\#3 (Srinivasan et al., 2012). However, the finding that icas\#3-mediated attraction and aggregation are not reduced by high NPR-1 activity indicated that icas\#3 responses involve signaling pathways that are partially distinct from those integrating responses to non-indole ascarosides, e.g., ascr\#3, and other types of aggregation-inducing stimuli, e.g., low oxygen levels. This hypothesis was supported by the observation that hermaphrodites lacking the RMG interneuron, which coordinates other aggregation responses via NPR-1, were still attracted to icas\#3 (Srinivasan et al., 2012).

In summary, indole ascarosides function as highly potent aggregation pheromones. Whether hermaphrodite attraction to indole ascarosides is additive or synergistic, as is the case for non-indole ascarosides in promoting dauer or male attraction, has not been investigated. Additional aggregation-inducing ascarosides, of which some exceed the indole ascarosides' in potency, have recently been identified via mass spectrometry-based comparative metabolomics (von Reuss et al., 2012). For example, the $p$-hydroxy benzoyl ascaroside hbas\#3 (Figure 8) attracts hermaphrodites at concentrations as low as $10 \mathrm{fM}$ (femtomolar) (von Reuss et al., 2012).<smiles>CC1C[C@H](OC(=O)c2ccc(O)cc2)[C@H](O)[C@H](O)C(O[C@@H](C)CCCC/C=C/C(=O)O)O1</smiles>

Figure 8. Structure of the hermaphrodite attractant hbas\#3 (von Reuss et al., 2012). 


\subsection{Male-produced ascarosides attract hermaphrodites}

Ascaroside production has been studied mostly by using mixed-gender cultures, which effectively profiled ascaroside production in hermaphrodites which account for more than $99 \%$ of wild-type worms under typical lab conditions (Chasnov and Chow, 2002). Izrayelit et al. showed that male C. elegans also produce ascarosides and that their ascaroside profiles differ starkly from those of hermaphrodites (Izrayelit et al., 2012). Male ascaroside profiles are dominated by the saturated ascr\#10, whereas hermaphrodites produce the corresponding $\alpha, \beta$-unsaturated ascaroside, ascr\#3, as major component. This seemingly minor difference of chemical structures (Figure 9) is associated with profound differences in biological activity. Whereas hermaphrodite-produced ascr\#3 repels hermaphrodites and attracts males, male-produced ascr\#10 strongly retains and attracts hermaphrodites. Hermaphrodites respond to extremely low quantities of ascr\#10 and it appears that the amounts of ascr\#10 excreted by a single male may be sufficient to affect hermaphrodite behavior. The observed differences between male and hermaphrodite ascaroside production appear to result from sex-specific expression of specific components of peroxisomal $\beta$-oxidation (the role of peroxisomal $\beta$-oxidation in ascaroside biosynthesis is discussed in Section 4).

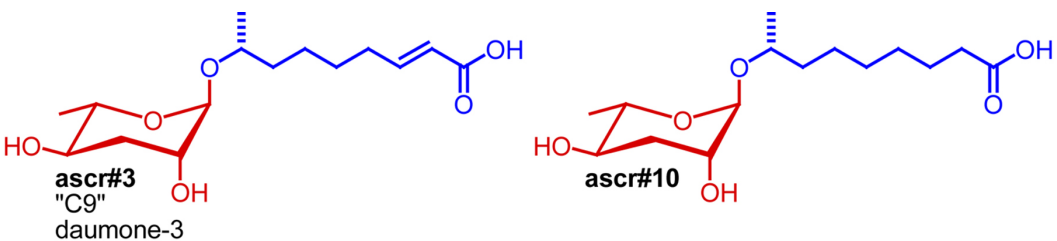

Figure 9. Structures of hermaphrodite-repelling ascr\#3 and the closely related, hermaphrodite-attracting ascr\#10 (Izrayelit et al., 2012 ).

\subsection{Ascaroside exposure affects olfactory plasticity}

In addition to directly affecting worm behavior, previous ascaroside exposure affects chemotaxis responses to other odorants. Yamada et al. noted that wild-type worms' response to the odorant benzaldehyde depends on population density (Yamada et al., 2010). For worms raised at low population densities, benzaldehyde remained attractive even after pre-exposure to this odorant, whereas for worms grown at higher population density pre-exposure to benzaldehyde resulted in loss of attraction. Notably, this plasticity of olfactory responses was absent in daf-22 worms, which are defective in ascaroside biosynthesis, suggesting that ascaroside exposure enhances olfactory plasticity. This hypothesis was confirmed by addition of natural dauer pheromone or synthetic ascr\#2, ascr\#3, or ascr\#5, which restored olfactory plasticity to daf-22 mutant worms. Dauer pheromone and synthetic ascarosides downregulate expression of a signaling peptide, SNET-1, that negatively regulates olfactory plasticity (Yamada et al., 2010). These results provide evidence for small-molecule conditioning of developing worms (or their progenitors) and connect population density signaling via ascarosides with endogenous peptide signaling that regulates chemotactic behavior. Similarly, earlier work by Peckol et al. had shown that exposure to natural dauer pheromone increases expression of three predicted chemosensory receptor genes $s r d-1$, str-2, and str-3, in the ASI chemosensory neurons, suggesting that experience-dependent change in chemosensory receptor gene expression may modify olfactory behaviors (Peckol et al., 2001).

\subsection{Ascaroside nomenclature}

Following the identification of the first ascaroside-based signaling molecules, compounds were named simply by referring to the lengths of their side chain, e.g., the 6-carbon ascr\#2 was named "C6" and the 9-carbon ascr\#3 was named "C9" (Butcher et al., 2007). In another naming system, "daumone-1", "daumone-2", and "daumone-3" have been used to refer to the dauer-inducing ascr\#1, ascr\#2, and ascr\#3 (Jeong et al., 2009). With the identification of now more than 100 additional ascarosides these naming systems became impractical (von Reuss et al., 2012). For example, there are at least six different simple ascarosides that feature a 9-carbon side chains: ascr\#3, ascr\#10, oscr\#3, oscr\#10, bhas\#10, and bhos\#10, making the name "C9" inconvenient and potentially misleading (Figure 10). Furthermore, names such as "C3", "C6", and "C9" are unsuitable for text mining. Lastly, a large number of modified ascarosides featuring indole-3-carboxy, $p$-hydroxybenzoyl, 2-methyl-2-butenoyl, glucosyl, $p$-aminobenzoyl moieties have been identified for which simple, unambiguous names must be found (von Reuss et al., 2012). 

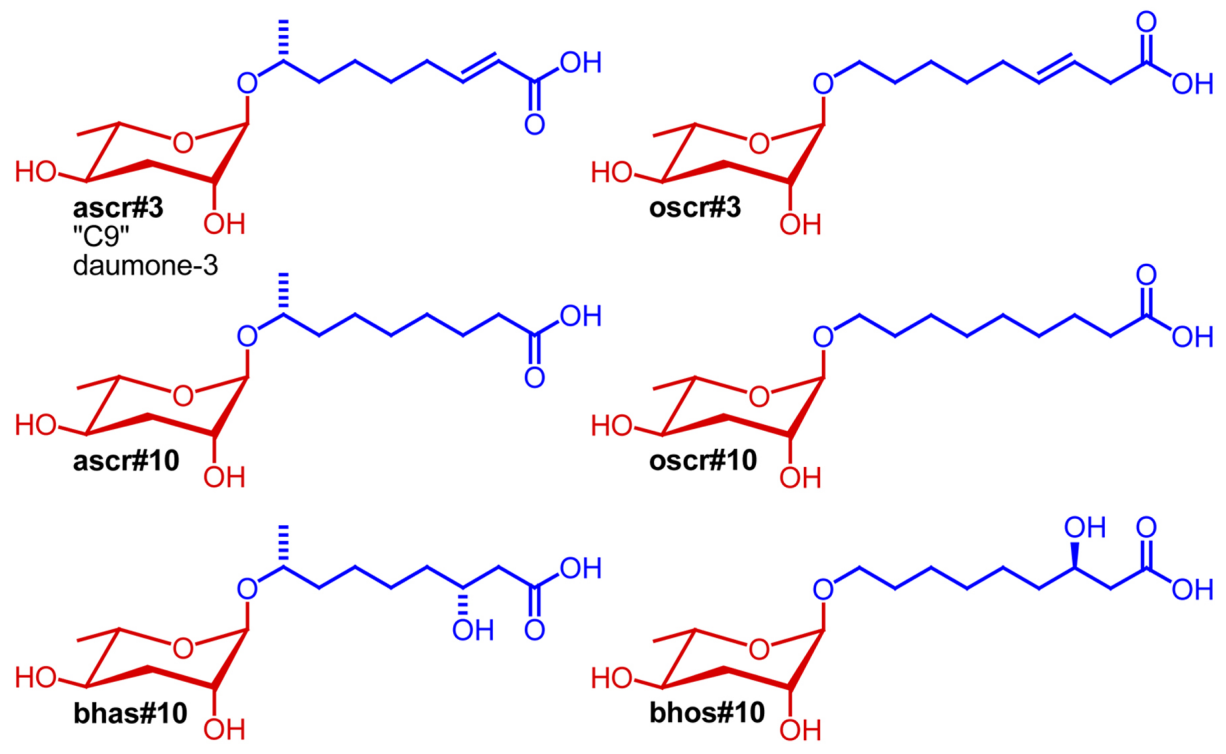

Figure 10. Structures of six simple ascarosides with 9-carbon side chains (Butcher et al., 2007; Srinivasan et al., 2012; von Reuss et al., 2012).

Therefore, a new naming system was developed in collaboration with WormBase (Srinivasan et al., 2012; von

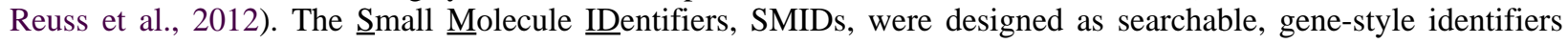
consisting of four lower case non-italicized letters that refer to the general structural class of the compound, followed by a pound sign and a number, e.g., ascr\#3, icas\#7, or hbas\#10. In collaboration with WormBase, SMIDs will be assigned for all small molecules newly identified from $C$. elegans and other nematodes. The SMID database (www.smid-db.org) serves as a publicly available reference for all assigned SMIDs. SMID-DB provides chemical structures, molecular formulas, a full list of references and other data for all SMIDs.

\section{Receptors}

C. elegans perceives environmental cues via specified neurons, which initiate signaling cascades triggering a wide variety of physiological responses. As described above, ascarosides affect larval development, aggregation, and mating behavior. This section summarizes current knowledge of the mechanisms of ascaroside perception.

The $C$. elegans genome encodes a very large number of G protein-coupled receptors (GPCRs). Expression studies suggest that of the more than 1000 predicted GPCRs, a majority are expressed exclusively or predominantly in the chemosensory neurons (Bargmann, 2006). The involvement of GPCRs in ascaroside perception has been suggested by the finding that upregulation of G protein alpha subunits gpa-2 and gpa-3 induces dauer formation, whereas gpa-2 and gpa-3 mutants are Daf-d. Similarly, putative guanylyl cyclase odr-1 mutants have reduced sensitivity to dauer pheromone, indicating a role in ascaroside reception. (Lans and Jansen, 2007; Zwaal et al., 1997).

At least five different types of chemosensory neurons are associated with dauer formation, the ADF, ASG, ASI, ASJ, and, to a lesser extent, ASK neurons (Bargmann, 2006), whereas male mating behavior and ascaroside-mediated hermaphrodite behaviors have been shown to involve the ASK, ASI, and ADL neurons (Jang et al., 2012; Macosko et al., 2009; Srinivasan et al., 2008; Srinivasan et al., 2012; White and Jorgensen, 2012) (Figure 11). This suggested that each of these neurons express one or several GPCRs that mediate ascaroside perception. 


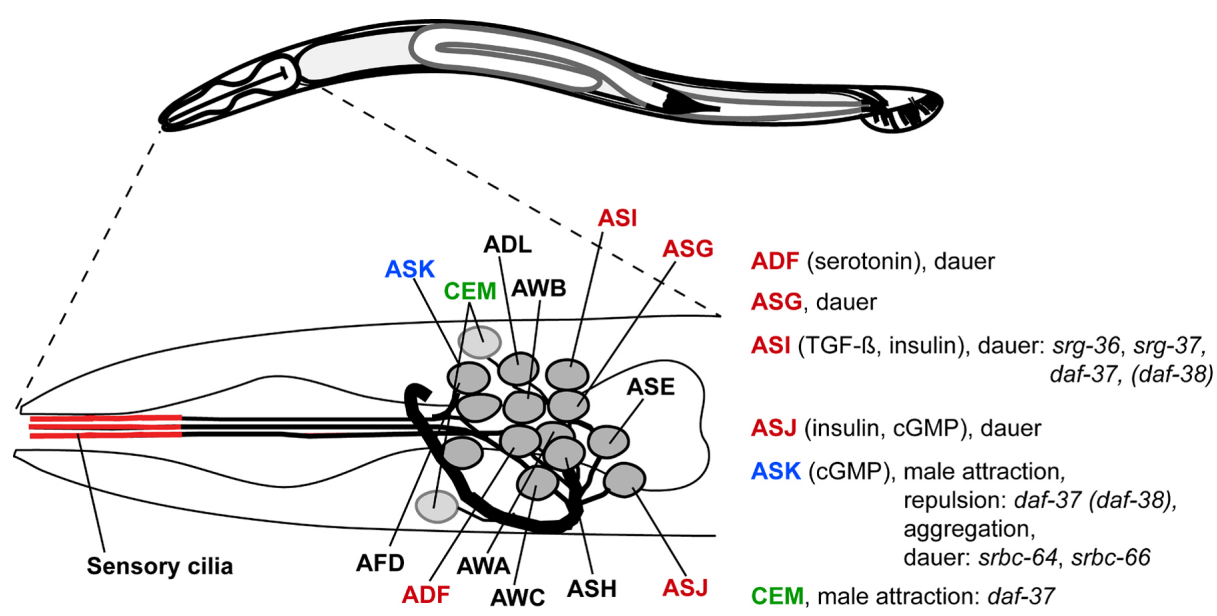

Figure 11. Head neurons (including the male-specific CEM neurons), their roles for ascaroside-mediated phenotypes, downstream signaling pathways, and so-far identified receptors (adapted from http://www.wormatlas.org).

Using a screen for mutants exhibiting altered responses to dauer pheromone, Kim et al. identified the first two GPCRs involved in ascaroside perception (Kim et al., 2009). Loss-of-function mutations in $s r b c-64$ and the closely related srbc-66, members of a large subfamily of Caenorhabditis-specific GPCRs, resulted in reduced dauer formation in response to natural dauer pheromone and synthetic ascarosides ascr\#1, ascr\#2, and ascr\#3. In contrast, dauer formation in response to synthetic ascr\#5 was less affected. Mutations in another closely related GPCR, $s r b c-65$, do not affect sensitivity to pheromone components. Kim et al. found that $s r b c-64$ and $s r b c-66$ are expressed in the ASK neurons, where they localize to the sensory cilia, but not the ASI neurons. This finding was surprising given that dauer pheromone exerts its effects primarily via the ASI neurons whereas the ASK neurons are thought to play a comparatively lesser role in dauer formation. However, expression of $s r b c-64$ or $s r b c-66$ under the control of an ASK-specific promoter fully rescued dauer-defective phenotypes of $s r b c-64$ or $s r b c-66$ mutants. Heterologous co-expression of $s r b c-64$ or $s r b c-66$ in HEK393 cells enhanced forskolin-mediated increases in cAMP levels, which could be suppressed by addition of ascr\#2 or ascr\#3, further supporting the role of $s r b c-64$ or $s r b c-66$ as ascaroside receptors. However, srbc-64; srbc-66 double mutants as well as the single mutants still formed dauer larvae in response to high concentrations of ascr\#1, ascr\#2, and ascr\#3, suggesting that additional GPCRs are involved in perception of these ascarosides.

The first ascr\#5-specific receptors were identified by McGrath et al. who investigated multigenic resistance to pheromone-induced dauer formation in two laboratory strains, LSJ2 and CC1, that had been grown for 4 years and 50 years in liquid axenic media (McGrath et al., 2011). Both strains do not form dauer larvae in response to dauer pheromone obtained from wild-type (N2) worms or synthetic samples of four main components of the dauer pheromone, ascr\#2, ascr\#3, ascr\#5, and icas\#9. By using recombinant inbred lines between a dauer defective strain and N2 worms for quantitative trait locus mapping, McGrath et al. identified two predicted GPCR's, srg-36 and $s r g-37$, in the X chromosome region associated with ascr\#5 resistance. Notably, $\mathrm{srg}$-36 and $\mathrm{srg}$ - 37 were found to be disrupted in both ascr\#5-resistant strains. Introgression of the genomic region containing the $\mathrm{srg}$-36 and $\mathrm{srg}$-37 deletion into the $\mathrm{N} 2$ background conferred resistance to ascr\#5, but did not significantly affect sensitivity to other ascarosides, indicating that $\mathrm{srg}-36$ and $\mathrm{srg}-37$ are specifically required for perception of ascr\#5. Complementing ascr\#5-resistant strains with either $\mathrm{srg}$-36 and $\mathrm{srg}$-37 cDNAs restored sensitivity to ascr\#5, indicating that the $\mathrm{srg}$-36 and $\operatorname{srg}-37$ genes are at least partially redundant. Both $s r g-36$ and $\operatorname{srg}-37$ are strongly expressed in the ASI neurons where they localize to the sensory cilia, consistent with a function as dauer pheromone receptors. McGrath et al. further showed that multigenic resistance to dauer pheromone has arisen also in C. briggsae, resulting in part from mutation of a paralog of $\mathrm{srg}$-36 and $\mathrm{srg}$-37 (McGrath et al., 2011).

Two additional ascaroside receptors, $d a f-37$ and $d a f-38$, were identified by Park et al. among proteins pulled down via DAF-8 immunoprecipitation (Park et al., 2012). daf-37 mutants are defective in all responses to ascr\#2 but respond normally to other ascarosides. In contrast, daf-38 mutants are partially defective in responses to several different ascarosides, including ascr\#2, ascr\#3, and ascr\#5. Photo-affinity labeling studies showed that DAF-37-dependent activity of ascr\#2 results from direct binding of the ascaroside to this GPCR, representing the first demonstration of direct molecular interactions between an ascaroside and a GPCR. Cell-specific overexpression showed that expression of DAF-37 in the ASI neurons mediates dauer formation whereas expression of DAF-37 in the ASK neurons regulates adult behavior. pbFRET (photo-bleaching Fluorescent Energy Transfer) assays revealed 
that the ascr\#2-specific DAF-37 forms heterodimers with DAF-38, and it was further shown that heterodimerization likely plays an important role for ascr\#2-perception (Park et al., 2012). The authors suggest that the ascarosides' synergistic and partially overlapping activity profiles may in part result from interaction of both structure-specific and more structure-promiscuous GPCRs, as several recent studies indicate that GPCRs often function as dimers of higher order oligomers (Bai, 2004; Milligan, 2010; Terrillon and Bouvier, 2004).

The great number of dauer pheromone components and the presence of receptors with redundant and overlapping functions indicates a highly sophisticated signaling system. The results of Kim et al., McGrath et al. and Park et al. show that ascaroside perception in nematodes involves at least three different families of GPCR's, some of which are highly specific, whereas others may be involved in perception of several different ascarosides (Kim et al., 2009; McGrath et al., 2011; Park et al., 2012). Whether srbc-64 and srbc-66 or srg-36 and srg-37 are also involved in mediating behavioral responses to ascarosides has not been studied in detail; however the observation that expression of $s r g-36 \mathrm{cDNA}$ or $s r g-37 \mathrm{cDNA}$ in the ASH neurons resulted in worms that respond strongly in an acute-avoidance assay suggests such a role for the $\mathrm{srg}$ genes (McGrath et al., 2011).

\section{Biosynthesis}

Shortly after the original description of the dauer pheromone in 1982, Golden and Riddle described a mutation, daf-22, that abolished dauer pheromone production (Golden and Riddle, 1985). Metabolite extracts derived from $d a f-22$ cultures failed to inhibit dauer recovery and were found to have little or no dauer inducing activity. Following the chemical identification of the dauer pheromone, it was shown that daf-22 worms do not produce the dauer inducing and male-attracting ascarosides ascr\#2 and ascr\#3 (Srinivasan et al., 2008). These results confirmed that ascaroside pheromones are worm-derived and not the result of bacterial metabolism, and also suggested a possible entry point for investigating ascaroside pheromone biosynthesis.

In 2006, Mak et al. reported a forward genetic screen for identifying fat-storage mutants that showed abnormal staining with C1-BODIPY-C12, a fluorescently labeled fatty acid analog that enabled monitoring fat storage and distribution in live worms (Mak et al., 2006). A subsequent study showed that a group of four of the identified fat storage mutants mapped to the same ORF as the canonical allele of daf-22 (Butcher et al., 2009). Sequence analysis revealed that daf-22 encodes a thiolase that is homologous to the mammalian peroxisomal 3-ketoacyl-CoA thiolase SCPx, which participates in peroxisomal $\beta$-oxidation of straight-chain and branched-chain fatty acids and bile acids. A second group of fat storage mutants mapped to another gene, dhs-28, with strong sequence similarity to the dehydrogenase domain of mammalian peroxisomal multifunctional protein, which acts upstream of SCPx in mammalian peroxisomal $\beta$-oxidation. These results suggested that in $C$. elegans DHS-28 may act upstream of DAF-22 in peroxisomal $\beta$-oxidation and, given that $d a f-22$ worms do not produce dauer pheromone, both DAF-22 and DHS-28 partake in ascaroside pheromone biosynthesis. Notably, transgenic intestinal DAF-22 expression is sufficient to restore dauer inducing activity of $d a f-22$ mutants, implicating the intestine as a major source of ascaroside pheromones (Butcher et al., 2009). The precise roles of DHS-28, DAF-22 and other peroxisomal enzymes in the biosynthesis of the ascaroside side chains was characterized in detail using mass spectrometry-based comparative metabolomics (von Reuss et al., 2012).

\subsection{Ascaroside side chains originate from peroxisomal $\beta$-oxidation}

Peroxisomal $\beta$-oxidation is a 4-step process that shortens long-chain fatty acids (LCFA) and very long-chain fatty acids (VLCFA, chain length $>\mathrm{C}_{24}$ ) iteratively by two carbon units, producing acetyl-CoA that enters the citric acid cycle (Figure 12). Peroxisomal fatty acid $\beta$-oxidation is believed to cease at a chain length of about 6-12 carbons, with $\beta$-oxidation of shorter chained fatty acids proceeding in the mitochondria (Reddy and Hashimoto, 2001). In ascaroside biosynthesis, the acyl-CoA oxidase ACOX-1 participates in the first step of peroxisomal side chain $\beta$-oxidation, introducing $\alpha, \beta$-unsaturation of long-chained precursors (Joo et al., 2010; von Reuss et al., 2012). Mass spectrometry-based comparative metabolomics showed that in acox-1 mutants production of ( $\omega$-1)-functionalized ascarosides with less than 9 carbons was largely abolished (von Reuss et al., 2012). In addition, amounts of the 9-carbon $\alpha, \beta$-unsaturated ascr\#3, usually the most abundant ascaroside in the wild-type excretome, was greatly reduced. Instead large quantities of ascr\#10, the saturated derivative of ascr\#3 and a homologous series of longer-chained saturated ascarosides was observed (von Reuss et al., 2012). 
(a)

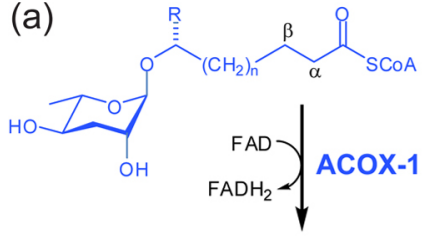

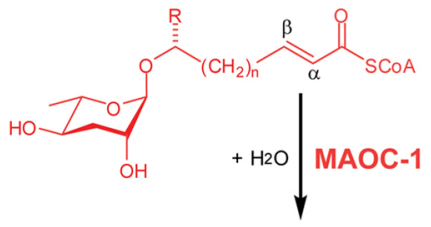

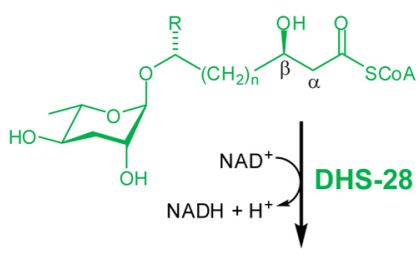

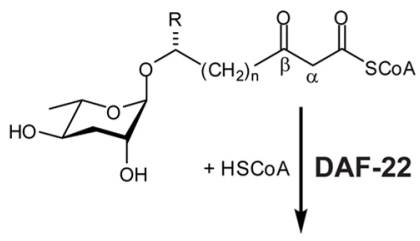

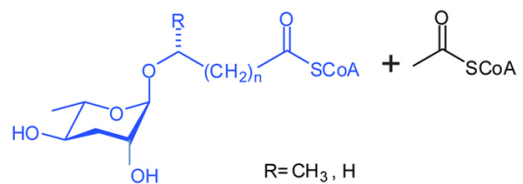

(b)
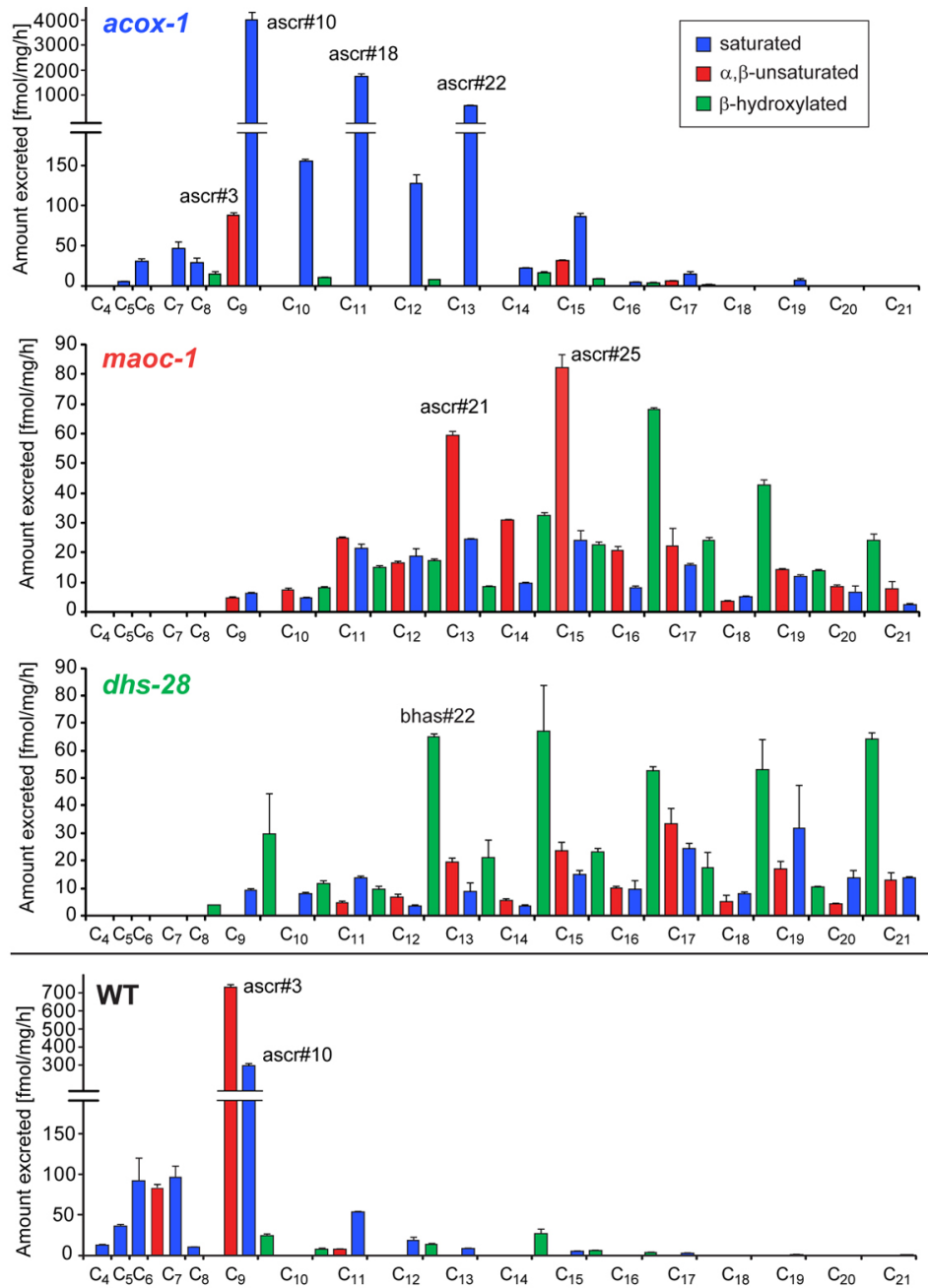

Figure 12. Ascaroside side-chain biosynthesis. (a) Proposed biosynthetic roles of peroxisomal $\beta$-oxidation enzymes ACOX-1, MAOC-1, DHS-28, and DAF-22. (b) ( $\omega$-1)-oxygenated ascarosides in wild-type and three $\beta$-oxidation mutants (acox-1, maoc-1, and dhs-28) with saturated (blue), $\alpha, \beta$-unsaturated (red), and $\beta$-hydroxylated (green) side chains. Adapted with permission from (von Reuss et al., 2012). Copyright 2012 American Chemical Society.

Notably, the acox-1 mutation affected biosynthesis of $(\omega)$-functionalized ascarosides differently than $(\omega-1)$-functionalized ascarosides. In acox-1 mutants, production of ascr\#5, the most abundant $(\omega)$-functionalized ascaroside in wild type, is completely abolished, whereas production of longer chain $(\omega)$-functionalized ascarosides with 5-13-carbon side chains, e.g., oscr\#9, is strongly increased. Thus in acox-1 mutants chain-shortening of $(\omega)$-functionalized ascarosides stalls at a chain length of 5 carbons whereas chain-shortening of ( $\omega$-1)-functionalized ascarosides ceases already at a chain length of 9 carbons. These results strongly suggest a model for ascaroside biosynthesis in which side-chain oxygenation, and perhaps ascarylose attachment, precedes peroxisomal $\beta$-oxidation (von Reuss et al., 2012). Because biosynthesis of short and medium-chained ascarosides is not completely abolished in acox-1 loss-of-function mutants, it is assumed that other, yet unidentified acyl-CoA oxidases participate in ascaroside biosynthesis (Joo et al., 2010; von Reuss et al., 2012). Furthermore, these results implicate ACOX-1 in peroxisomal $\beta$-oxidation of short-chain fatty acids, in contrast to the notion that $\beta$-oxidation of short chain fatty acids occur primarily in the mitochondria (Reddy and Hashimoto, 2001).

Whereas in vertebrates and Drosophila the next two steps of iterative peroxisomal chain shortening, hydration of the double bond and subsequent dehydrogenation to the $\beta$-ketoacyl-CoA ester, are catalyzed by one protein, e.g., MFE-2, these two enzymatic functions are associated with two separate proteins in $C$. elegans, MAOC- 1 and DHS-28 (Figure 12) (Haataja et al., 2011; von Reuss et al., 2012; Zhang et al., 2010). Hydration of the double bond in the $\alpha, \beta$-unsaturated side chains produced by ACOX-1 is catalyzed by the peroxisomal 2-enoyl-CoA hydratase MAOC-1. Correspondingly, maoc-1 loss-of-function mutants accumulate medium and long-chain $\alpha, \beta$-unsaturated ascarosides, along with smaller amounts of saturated and $\beta$-hydroxylated derivatives. In contrast to acox- 1 mutants, 
biosynthesis of short-chain ascarosides dominant in wild-type worms is completely abolished in maoc- 1 worms (von Reuss et al., 2012).

Similarly, wild-type ascarosides are completely absent from the $d h s-28$ and daf-22 metabolomes (Butcher et al., 2009; von Reuss et al., 2012). Comparative metabolomic analyses of $d h s-28$ metabolome samples revealed build-up of $\beta$-hydroxylated medium- and long-chain ascarosides, which likely derive from corresponding $\beta$-hydroxyacyl-CoA derivatives that are the substrates of the $\beta$-hydroxyacyl-CoA dehydrogenase DHS-28 (Figures 12 and 13). In addition, $d h s-28$ worms produce smaller amounts of medium- and long-chain saturated and $\alpha, \beta$-unsaturated ascarosides. daf-22 worms produce only very small amounts of medium- and long-chain ascarosides, most of which have saturated or $\beta$-hydroxylated side chains (von Reuss et al., 2012). Some of these long-chain ascarosides have been shown to have weak dauer inducing activity and may account for the small amount of dauer-inducing activity occasionally present in $d h s-28$ and daf-22 metabolite extracts (Butcher et al., 2009; von Reuss et al., 2012). daf-22 worms additionally contain larger quantities of very long chain ascarosides similar to the ascaroside lipids identified from Ascaris egg shells (Bartley et al., 1996; Jezyk and Fairbairn, 1967; Pungaliya et al., 2009). These very long-chain ascarosides may represent the ultimate precursors of all or some of the ascarosides described in this section.
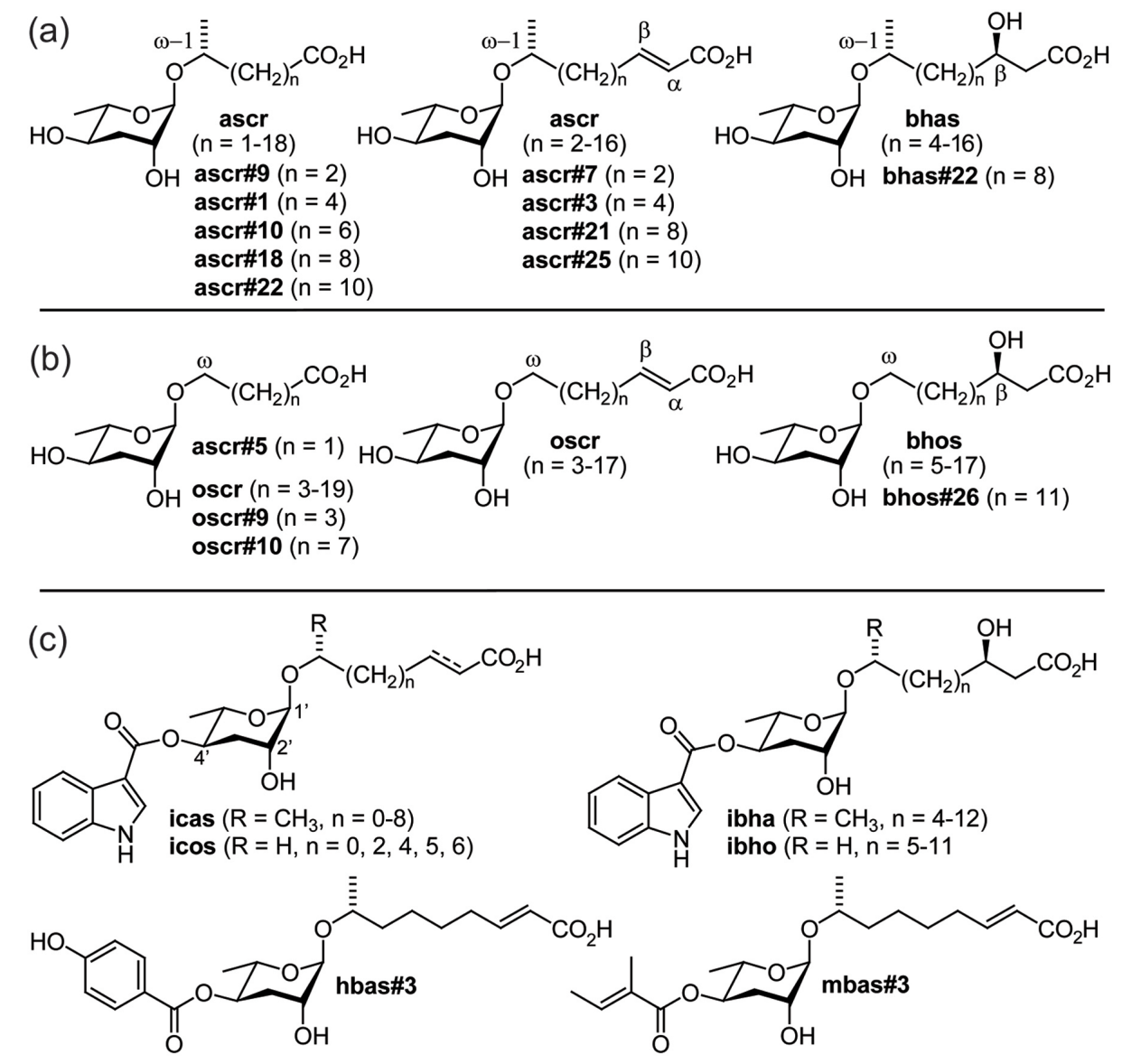

Figure 13. Ascarosides identified in wild-type, acox-1, maoc-1, dhs-28, and daf-22 worms via LC-MS/MS. (a) ( $\omega$-1)-oxygenated ascarosides, (b) $(\omega)$-oxygenated ascarosides, and (c) examples for 4'-acylated derivatives. Reprinted with permission from (von Reuss et al., 2012 ). Copyright 2012 American Chemical Society.

Comparison on the ascaroside profiles of males and hermaphrodites indicates that peroxisomal $\beta$-oxidation in C. elegans is regulated in a sex-specific manner (Izrayelit et al., 2012). Male ascaroside profiles are dominated by ascr\#10, whereas hermaphrodites produce large amounts of ascr\#3, distinguished from ascr\#10 solely by the incorporation of a double bond, instead. These differences in ascaroside profiles correlate with sex-specific differences in the expression levels of peroxisomal $\beta$-oxidation genes. 


\subsection{Biosynthesis of indole ascarosides}

Short-chain indole ascarosides are absent from the maoc-1, dhs-28, and daf-22 mutant metabolomes, indicating that biosynthesis of the side chains of indole ascarosides and non-indole ascarosides proceeds via the same pathway (von Reuss et al., 2012). Correspondingly, acox-1, maoc-1, and $d h s-28$ worms produce medium- and long-chain indole ascarosides with saturated, $\alpha, \beta$-unsaturated, and $\beta$-hydroxylated side chains similar to those of the non-indole ascarosides found in these mutants (von Reuss et al., 2012). Using worms grown in axenic cultures, the indole carboxy moiety in wild-type indole ascarosides was shown to be derived from tryptophan (Srinivasan et al., 2012), and incubation of ascr\#3 with daf-22 worms showed that non-indole ascarosides serve as precursors for indole ascarosides (von Reuss et al., 2012). Similarly, p-hydroxybenzoyl ascarosides (e.g., hbas\#3) and (E)-2-methyl-2-butenyl ascarosides (e.g., mbas\#3) could be derived from the amino acids tyrosine and isoleucine, respectively. Comparison of the relative amounts of indole and non-indole ascarosides in wild-type worms indicates that indole attachment is tightly regulated. For example, ascr\#9 is much less abundant than ascr\#3, whereas the corresponding indole ascarosides icas\#9 and icas\#3 are of roughly equal abundance (von Reuss et al., 2012). Given that conversion of ascarosides into their indole carboxy derivatives is associated with a dramatic change in biological activity (see Section 2.4), it seems likely that specific mechanisms control indole ascaroside formation. However, enzymes catalyzing attachment of the indole carboxy moiety have not been identified.

\subsection{Ascarylose biosynthesis, side-chain functionalization, and a preliminary model}

Whereas the biosynthesis of dideoxysugar L-ascarylose in bacteria is well understood (Thorson et al., 1994), ascarylose biosynthesis in $C$. elegans has not been investigated in detail. Detection of ascarosides in axenic $C$. elegans liquid cultures demonstrated that worms produce ascarylose endogenously (Srinivasan et al., 2012), and the C. elegans genome includes several homologues of bacterial genes in this pathway (von Reuss et al., 2012), for example, ascE from Yersinia pseudotuberculosis (Thorson et al., 1994). Similarly, enzymes involved in hydroxylation and glycosylation of the VLCFA precursors of the ascaroside side-chains remain to be identified. Taken together, current knowledge allows proposing a preliminary model for ascaroside biosynthesis (Figure 14).

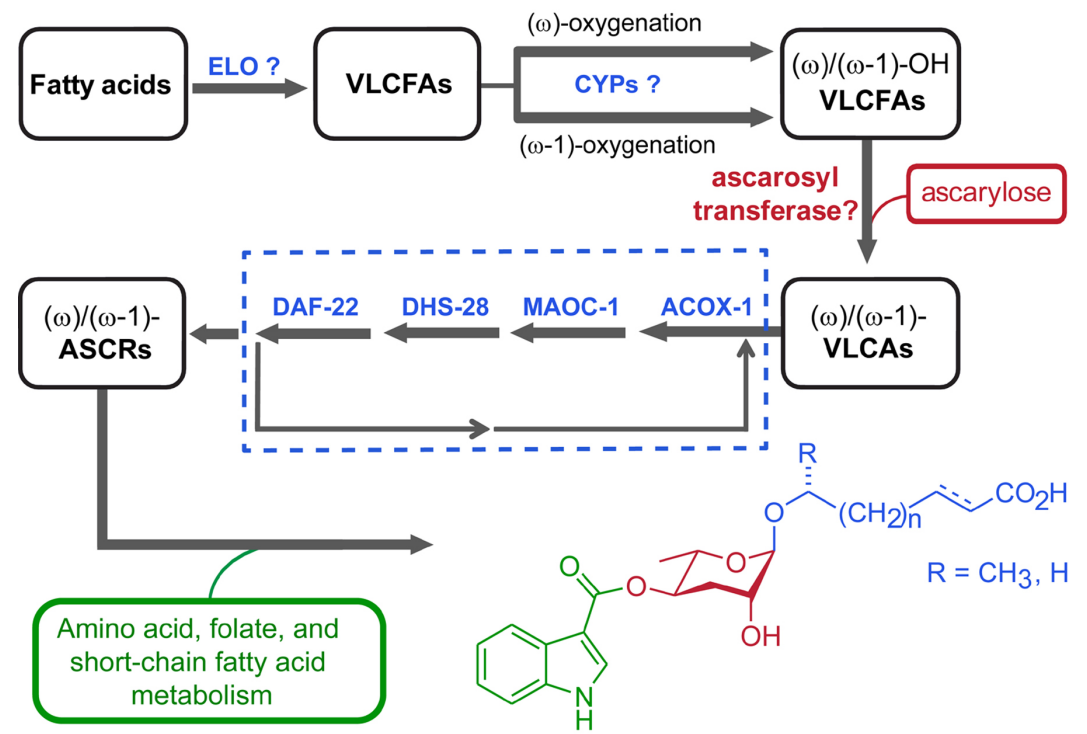

Figure 14. Model for ascaroside biogenesis. Chain elongation of fatty acids (by putative elongase homologs elo-1-9 (Agbaga et al., 2008)) is followed by $(\omega-1)$ - or $(\omega)$-oxygenation of VLCFAs and ascarylose attachment by yet unidentified enzymes. The resulting VLCAs enter peroxisomal $\beta$-oxidation via ACOX-1, MAOC-1, DHS-28, and DAF-22 producing short chain ascarosides, which are linked to building blocks from several different primary metabolic pathways. Adapted with permission from (von Reuss et al., 2012). Copyright 2012 American Chemical Society.

\subsection{Life stage and environmental conditions affect ascaroside production}

Production of ascaroside pheromones by C. elegans is stage dependent (Kaplan et al., 2011). Total amounts of ascarosides excreted increases throughout development from L1 to adult worms. Excretion of the two main components of the male-attracting signal, ascr\#3 and ascr\#8, peaks at the young adult stage (Kaplan et al., 2011). Under dauer-inducing conditions, excretion of the strongly dauer-inducing ascr\#2 peaks right before entry into the 
dauer stage. Dauer worms do not appear to excrete ascarosides, but contain small amounts of ascr\#1 and ascr\#3 (Kaplan et al., 2011).

Temperature affects the ratio of ascr\#1 and ascr\#3 production (Joo et al., 2010; Lee et al., 2010) as well as the amount of ascr\#5 excreted (Butcher et al., 2008). Comparison of the ascaroside profiles of well-fed and starved $C$. elegans wild-type cultures showed that starved worms excrete significantly larger amounts of the ( $\omega$-1)-linked ascr\#3 than well-fed worms, relative to the amounts of $\omega$-linked ascr\#5 (von Reuss et al., 2012). Similarly, analysis of $d h s-28$ mutant liquid cultures revealed that excretion of long-chain ( $\omega-1)$-oxygenated ascarosides in starved $d h s-28$ cultures was about 5 times higher than those of well-fed $d h s-28$ cultures (von Reuss et al., 2012). Since the $\omega$-linked ascr\#5 affects worm behavior differently than the major ( $\omega-1)$-linked ascarosides, e.g., ascr\#3 (Butcher et al., 2008; Macosko et al., 2009), it appears that ascaroside signaling is actively regulated in response to starvation via changes in the ratio of $(\omega-1)$ to $(\omega)$-functionalization of the VLCFA precursors of the ascaroside side chains (von Reuss et al., 2012). Increasingly sensitive analytical techniques will enable more detailed studies of ascaroside production of different life stages under different environmental conditions.

\subsection{Ascaroside excretion is selective}

Comparison of the ascaroside profiles of liquid culture supernatant extracts (worm excretome) and worm body extracts showed that ascaroside are differentially released (Kaplan et al., 2011; von Reuss et al., 2012). Saturated ascarosides are retained in the worm bodies to a much greater extent than their $\alpha, \beta$-unsaturated derivatives. In addition, worm bodies contain large quantities of ascaroside O-glycoside esters, for example the ascr\#10 glucoside glas\#10, which are absent from media supernatant samples (von Reuss et al., 2012).

\section{A modular library of signaling molecules}

As discussed in Section 2, ascaroside-based small-molecule signals regulate a stunningly diverse range of phenotypes, including dauer arrest, mate attraction, aggregation, and olfactory plasticity, and it is likely that ascarosides affect additional aspects of $C$. elegans life history, for example, adult lifespan and stress resistance. This wide variety of biological functions is mediated by a corresponding diversity of ascaroside structures, which are generated via modular assembly of building blocks from carbohydrate, lipid, short-chain fatty acid, amino acid, and folate metabolism. Therefore, it appears that ascaroside biosynthesis integrates input from several major metabolic pathways to encode and communicate comprehensive information about reproductive and nutritional state (von Reuss et al., 2012). More detailed investigation of ascaroside biosynthetic pathways and their regulation may provide insight into how information about metabolic and reproductive state as well as environmental parameters is transduced by ascaroside signaling.

Additional levels of complexity in ascaroside signaling arise from (1) the functioning of individual ascarosides in more than one pathway (e.g., ascr\#3 induces dauer, attracts males, and deters hermaphrodites), and (2) the ascarosides' synergistic modes of action (Figure 15). As evident from the complicated history of the dauer pheromone identification, the reconstitution of biological activity using synthetic compounds faces considerable challenges, not only because a large number of different compounds have to be synthesized, but also because the exact composition of chemical signals may often depend on many variables, including environmental conditions and the history of the pheromone-perceiving worms. Given the number of ascarosides identified so far and the great variety of phenotypes downstream of ascaroside signaling, elucidation of the mechanisms of ascaroside perception and signal integration remains a formidable task. 


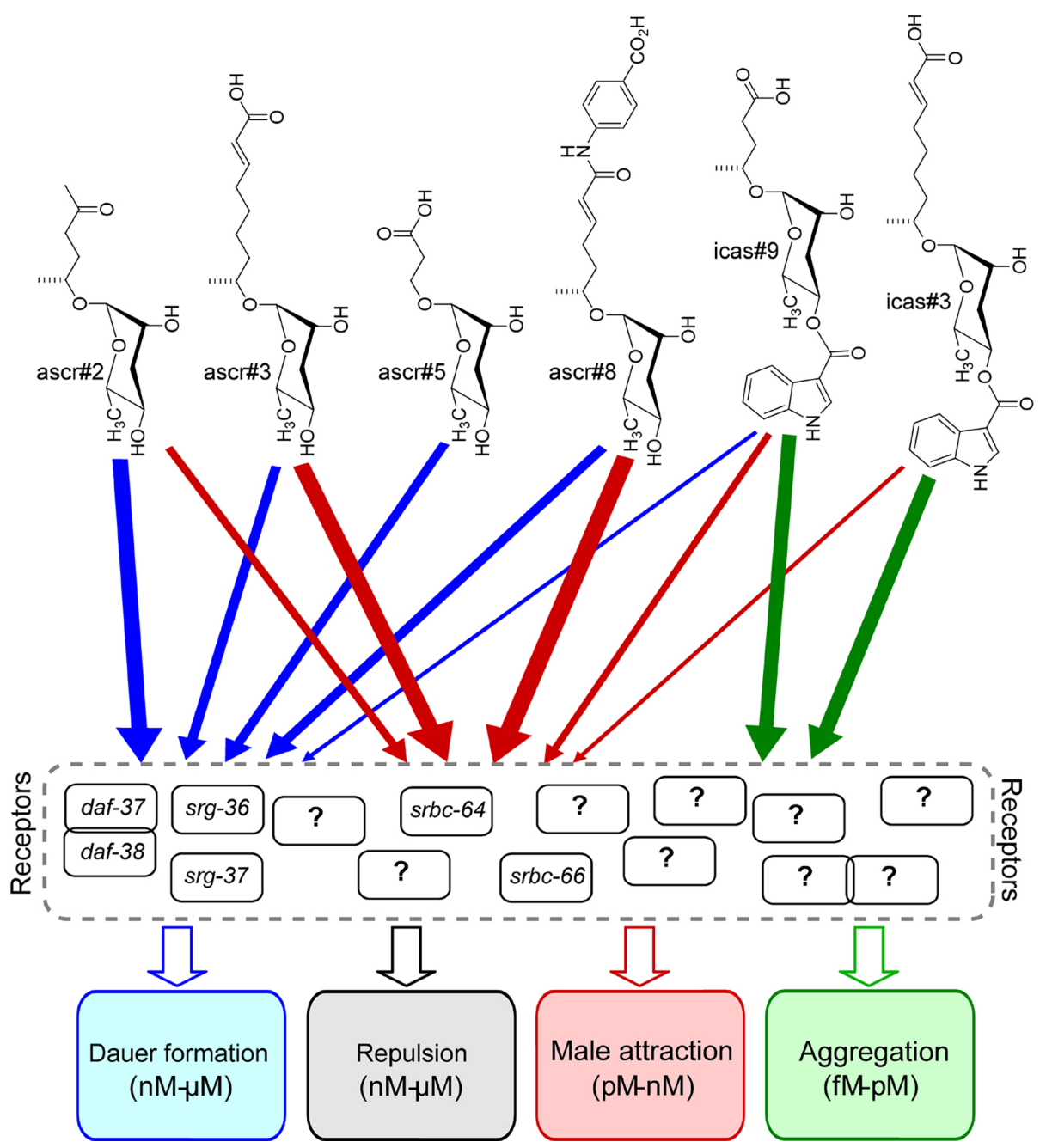

Figure 15. Synergistic blends of specific ascaroside combinations and concentrations elicit different phenotypes via activation of GPCR's in chemosensory neurons. The structures of most ascaroside receptors and their modes of action are not known.

Ascaroside signaling appears to be widely conserved among both free-living and parasitic nematode species (Choe et al., 2012b). In screening for the more than 100 different ascarosides so far identified, 15 out of 18 tested nematode species were found to produce ascarosides known from $C$. elegans. Curiously, the three tested species in which none of the C. elegans ascarosides could be detected included the pig parasite Ascaris suum, congeneric with A. lumbricoides in which long-chain ascarosides were first detected more than 100 years ago (Flury, 1912). Nonetheless, it is possible that $A$. suum does produce short-chain ascarosides similar to those identified from $C$. elegans and other nematode species, which may not have been detected due to their low abundance, stage-specific production, or unexpected structural features. More detailed analysis of C. elegans and other nematode species will likely reveal additional structural modifications of ascarosides, as suggested by the recent identification of ascaroside ethanolamides from Heterorhabditis bacteriophora (Noguez et al., 2012) and multiply side chain-hydroxylated ascarosides from Panagrellus redivivus (Choe et al., 2012a).

\section{Acknowledgement}

The authors thank Jagan Srinivasan (Worchester Polytechnic Institute) for a critical reading of the manuscript. This work was supported in part by the National Institutes of Health (GM088290 and GM085285) 


\section{References}

Agbaga, M. P., Brush, R. S., Mandal, M. N., Henry, K., Elliott, M. H., and Anderson, R. E. (2008). Role of Stargardt-3 macular dystrophy protein (ELOVL4) in the biosynthesis of very long chain fatty acids. Proc. Natl. Acad. Sci. U. S. A. 105, 12843-12848. Abstract Article

Albert, P. S., and Riddle, D. L. (1988). Mutants of Caenorhabditis elegans that form dauer-like larvae. Dev. Biol. 126, 270-293. Abstract Article

Antebi, A., Yeh, W. H., Tait, D., Hedgecock, E. M., and Riddle, D. L. (2000). daf-12 encodes a nuclear receptor that regulates the dauer diapause and developmental age in C elegans. Genes Dev. 14, 1512-1527. Abstract

Bai, M. (2004). Dimerization of G-protein-coupled receptors: roles in signal transduction. Cell. Signal. 16, 175-186. Abstract Article

Bargmann, C. I. (2006). Chemosensation in C. elegans, WormBook, ed. The C. elegans Research Community, WormBook, doi/10.1895/wormbook.1.123.1, http://www.wormbook.org. Abstract Article

Barr, M. M., and Garcia, L. R. (2006). Male mating behavior. WormBook, ed. The C. elegans Research Community, WormBook, doi/10.1895/wormbook.1.78.1, http://www.wormbook.org. Abstract Article

Bartley, J. P., Bennett, E. A., and Darben, P. A. (1996). Structure of the ascarosides from Ascaris suum. J. Nat. Prod. 59, 921-926. Abstract Article

Bethke, A., Fielenbach, N., Wang, Z., Mangelsdorf, D. J., and Antebi, A. (2009). Nuclear hormone receptor regulation of microRNAs controls developmental progression. Science 324, 95-98. Abstract Article

Butcher, R. A., Fujita, M., Schroeder, F. C., and Clardy, J. (2007). Small-molecule pheromones that control dauer development in Caenorhabditis elegans. Nat. Chem. Biol. 3, 420-422. Abstract Article

Butcher, R. A., Ragains, J. R., and Clardy, J. (2009). An indole-containing dauer pheromone component with unusual dauer inhibitory activity at higher concentrations. Org. Lett. 11, 3100-3103. Abstract Article

Butcher, R. A., Ragains, J. R., Kim, E., and Clardy, J. (2008). A potent dauer pheromone component in Caenorhabditis elegans that acts synergistically with other components. Proc. Natl. Acad. Sci. U. S. A. 105, 14288-14292. Abstract Article

Butcher, R. A., Ragains, J. R., Li, W., Ruvkun, G., Clardy, J., and Mak, H. Y. (2009). Biosynthesis of the Caenorhabditis elegans dauer pheromone. Proc. Natl. Acad. Sci. U. S. A. 106, 1875-1879. Abstract Article

Cassada, R. C., and Russell, R. L. (1975). The dauerlarva, a post-embryonic developmental variant of the nematode Caenorhabditis elegans. Dev. Biol. 46, 326-342. Abstract Article

Chasnov, J. R., and Chow, K. L. (2002). Why are there males in the hermaphroditic species Caenorhabditis elegans? Genetics 160, 983-994. Abstract

Chasnov, J. R., So, W. K., Chan, C. M., and Chow, K. L. (2007). The species, sex, and stage specificity of a Caenorhabditis sex pheromone. Proc. Natl. Acad. Sci. U. S. A. 104, 6730-6735. Abstract Article

Choe, A., Chuman, T., von Reuss, S. H., Dossey, A. T., Yim, J., Ajredini, R., Kolawa, A. A., Kaplan, F., Alborn, H. T., Teal, P. E., Schroeder, F. C., Sternberg, P. W., and Edison, A. S. (2012a). Sex-specific mating pheromones in the nematode Panagrellus redivivus. Proc. Natl. Acad. Sci. U. S. A. 109, 20949-20954. Abstract Article

Choe, A., von Reuss, S. H., Kogan, D., Gasser, R. B., Platzer, E. G., Schroeder, F. C., and Sternberg, P. W. (2012b). Ascaroside signaling is widely conserved among nematodes. Curr. Biol. 22, 772-780. Abstract Article

de Bono, M., and Bargmann, C. I. (1998). Natural variation in a neuropeptide Y receptor homolog modifies social behavior and food response in C elegans. Cell 94, 679-689. Abstract Article

Fairbairn, D. (1957). The biochemistry of Ascaris. Exp. Parasitol. 6, 491-554. Abstract Article

Fielenbach, N., and Antebi, A. (2008). C elegans dauer formation and the molecular basis of plasticity. Genes Dev. 22, 2149-2165. Abstract Article

Flury, F. (1912). On the chemistry and toxicology of ascarides. Arch. Exp. Path. Pharmak. 67, 275-392. Article

Foor, W. E. (1967). Ultrastructural aspects of oocyte development and shell formation in Ascaris lumbricoides. J. Parasitol. 53, 1245-1261. Abstract Article 
Fouquey, C., Polonsky, J., and Lederer, E. (1957). Uber die chemische Struktur der Ascarylose, des Zuckeranteils der Ascaroside. Angew. Chem., Int. Ed. Engl. 69, 679-679.

Gallo, M., and Riddle, D. L. (2009). Effects of a Caenorhabditis elegans dauer pheromone ascaroside on physiology and signal transduction pathways. J. Chem. Ecol. 35, 272-279. Abstract Article

Gerisch, B., Weitzel, C., Kober-Eisermann, C., Rottiers, V., and Antebi, A. (2001). A hormonal signaling pathway influencing $C$ elegans metabolism, reproductive development, and life span. Dev. Cell 1, 841-851. Abstract Article

Golden, J. W., and Riddle, D. L. (1982). A pheromone influences larval development in the nematode Caenorhabditis elegans. Science 218, 578-580. Abstract Article

Golden, J. W., and Riddle, D. L. (1984). The Caenorhabditis elegans dauer larva: developmental effects of pheromone, food, and temperature. Dev. Biol. 102, 368-378. Abstract Article

Golden, J. W., and Riddle, D. L. (1985). A gene affecting production of the Caenorhabditis elegans dauer-inducing pheromone. Mol. Gen. Genet. 198, 534-536. Abstract Article

Haataja, T. J., Koski, M. K., Hiltunen, J. K., and Glumoff, T. (2011). Peroxisomal multifunctional enzyme type 2 from the fruitfly: dehydrogenase and hydratase act as separate entities, as revealed by structure and kinetics. Biochem. J. 435, 771-781. Abstract Article

Hodgkin, J., and Doniach, T. (1997). Natural variation and copulatory plug formation in Caenorhabditis elegans. Genetics 146, 149-164. Abstract

$\mathrm{Hu}$, P. J. (2007). Dauer. WormBook, ed. The C. elegans Research Community, WormBook, doi/10.1895/wormbook.1.144.1, http://www.wormbook.org. Abstract Article

Izrayelit, Y., Srinivasan, J., Campbell, S. L., Jo, Y., von Reuss, S. H., Genoff, M. C., Sternberg, P. W., and Schroeder, F. C. (2012). Targeted metabolomics reveals a male pheromone and sex-specific ascaroside biosynthesis in Caenorhabditis elegans. ACS Chem. Biol. 7, 1321-1325. Abstract Article

Jang, H., Kim, K., Neal, S. J., Macosko, E., Kim, D., Butcher, R. A., Zeiger, D. M., Bargmann, C. I., and Sengupta, P. (2012). Neuromodulatory state and sex specify alternative behaviors through antagonistic synaptic pathways in $C$ elegans. Neuron 75, 585-592. Abstract Article

Jeong, P. Y., Jung, M., Yim, Y. H., Kim, H., Park, M., Hong, E., Lee, W., Kim, Y. H., Kim, K., and Paik, Y. K. (2005). Chemical structure and biological activity of the Caenorhabditis elegans dauer-inducing pheromone. Nature 433, 541-545. Abstract Article

Jeong, P. Y., Kwon, M. S., Joo, H. J., and Paik, Y. K. (2009). Molecular time-course and the metabolic basis of entry into dauer in Caenorhabditis elegans. PLoS One 4, e4162. Abstract Article

Jezyk, P. F., and Fairbairn, D. (1967). Ascarosides and ascaroside esters in Ascaris lumbricoides (Nematoda). Comp. Biochem. Physiol. 23, 691-705. Abstract Article

Joo, H. J., Kim, K. Y., Yim, Y. H., Jin, Y. X., Kim, H., Kim, M. Y., and Paik, Y. K. (2010). Contribution of the peroxisomal acox gene to the dynamic balance of daumone production in Caenorhabditis elegans. J. Biol. Chem. 285, 29319-29325. Abstract Article

Kaplan, F., Srinivasan, J., Mahanti, P., Ajredini, R., Durak, O., Nimalendran, R., Sternberg, P. W., Teal, P. E., Schroeder, F. C., Edison, A. S., and Alborn, H. T. (2011). Ascaroside expression in Caenorhabditis elegans is strongly dependent on diet and developmental stage. PLoS ONE 6, e17804. Abstract Article

Kim, K., Sato, K., Shibuya, M., Zeiger, D. M., Butcher, R. A., Ragains, J. R., Clardy, J., Touhara, K., and Sengupta, P. (2009). Two chemoreceptors mediate developmental effects of dauer pheromone in C elegans. Science 326, 994-998. Abstract Article

Klass, M., and Hirsh, D. (1976). Non-ageing developmental variant of Caenorhabditis elegans. Nature 260, 523-525. Abstract Article

Lans, H., and Jansen, G. (2007). Multiple sensory G proteins in the olfactory, gustatory and nociceptive neurons modulate longevity in Caenorhabditis elegans. Dev. Biol. 303, 474-482. Abstract Article

Larsen, P. L., Albert, P. S., and Riddle, D. L. (1995). Genes that regulate both development and longevity in Caenorhabditis elegans. Genetics 139, 1567-1583. Abstract

Lee, J., Kim, K. Y., Lee, J., and Paik, Y. K. (2010). Regulation of dauer formation by O-GlcNAcylation in Caenorhabditis elegans. J. Biol. Chem. 285, 2930-2939. Abstract Article 
Lee, S. S., and Schroeder, F. C. (2012). Steroids as central regulators of organismal development and lifespan. PLoS Biol. 10, e1001307. Abstract Article

Ludewig, A. H., Kober-Eisermann, C., Weitzel, C., Bethke, A., Neubert, K., Gerisch, B., Hutter, H., and Antebi, A. (2004). A novel nuclear receptor/coregulator complex controls $C$ elegans lipid metabolism, larval development, and aging. Genes Dev. 18, 2120-2133. Abstract Article

Macosko, E. Z., Pokala, N., Feinberg, E. H., Chalasani, S. H., Butcher, R. A., Clardy, J., and Bargmann, C. I. (2009). A hub-and-spoke circuit drives pheromone attraction and social behaviour in C elegans. Nature 458, 1171-1175. Abstract Article

Mak, H. Y., Nelson, L. S., Basson, M., Johnson, C. D., and Ruvkun, G. (2006). Polygenic control of Caenorhabditis elegans fat storage. Nat. Genet. 38, 363-368. Abstract Article

McGrath, P. T., Xu, Y., Ailion, M., Garrison, J. L., Butcher, R. A., and Bargmann, C. I. (2011). Parallel evolution of domesticated Caenorhabditis species targets pheromone receptor genes. Nature 477, 321-325. Abstract Article

Milligan, G. (2010). The role of dimerisation in the cellular trafficking of G-protein-coupled receptors. Curr. Opin. Pharmacol. 10, 23-29. Abstract Article

Motola, D. L., Cummins, C. L., Rottiers, V., Sharma, K. K., Li, T., Li, Y., Suino-Powell, K., Xu, H. E., Auchus, R. J., Antebi, A., and Mangelsdorf, D. J. (2006). Identification of ligands for DAF-12 that govern dauer formation and reproduction in Celegans. Cell 124, 1209-1223. Abstract Article

Noguez, J. H., Conner, E. S., Zhou, Y., Ciche, T. A., Ragains, J. R., and Butcher, R. A. (2012). A novel ascaroside controls the parasitic life cycle of the entomopathogenic nematode Heterorhabditis bacteriophora. ACS Chem. Biol. 7, 961-966. Abstract Article

Park, D., O'Doherty, I., Somvanshi, R. K., Bethke, A., Schroeder, F. C., Kumar, U., and Riddle, D. L. (2012). Interaction of structure-specific and promiscuous G-protein-coupled receptors mediates small-molecule signaling in Caenorhabditis elegans. Proc. Natl. Acad. Sci. U. S. A. 109, 9917-9922. Abstract Article

Peckol, E. L., Troemel, E. R., and Bargmann, C. I. (2001). Sensory experience and sensory activity regulate chemosensory receptor gene expression in Caenorhabditis elegans. Proc. Natl. Acad. Sci. U. S. A. 98, 11032-11038. Abstract Article

Pungaliya, C., Srinivasan, J., Fox, B. W., Malik, R. U., Ludewig, A. H., Sternberg, P. W., and Schroeder, F. C. (2009). A shortcut to identifying small molecule signals that regulate behavior and development in Caenorhabditis elegans. Proc. Natl. Acad. Sci. U. S. A. 106, 7708-7713. Abstract Article

Reddy, J. K., and Hashimoto, T. (2001). Peroxisomal $\beta$-oxidation and peroxisome proliferator-activated receptor $\alpha$ : an adaptive metabolic system. Annu. Rev. Nutr. 21, 193-230. Abstract Article

Riddle, D. L., and Albert, P. S. (1997). Genetic and environmental regulation of dauer larva development. In $C$ elegans II, D. L. Riddle, T. Blumenthal, B. J. Meyer, and J. R. Priess, eds. (Cold Spring Harbor (NY)). Abstract

Riddle, D. L., Swanson, M. M., and Albert, P. S. (1981). Interacting genes in nematode dauer larva formation. Nature 290, 668-671. Abstract Article

Rogers, C., Reale, V., Kim, K., Chatwin, H., Li, C., Evans, P., and de Bono, M. (2003). Inhibition of Caenorhabditis elegans social feeding by FMRFamide-related peptide activation of NPR-1. Nat. Neurosci. 6, 1178-1185. Abstract Article

Schaedel O.N., Gerisch B., Antebi A., and Sternberg P. W. (2012). Hormonal signal amplification mediates environmental conditions during development and controls an irreversible commitment to adulthood. PLoS Biol. 10, e1001306. Abstract Article

Schulz, F. N., and Becker, M. (1933). Ascaryl alcohol. Biochem. Z. 265, 253-259.

Simon, J. M., and Sternberg, P. W. (2002). Evidence of a mate-finding cue in the hermaphrodite nematode Caenorhabditis elegans. Proc. Natl. Acad. Sci. U. S. A. 99, 1598-1603. Abstract Article

Srinivasan, J., Kaplan, F., Ajredini, R., Zachariah, C., Alborn, H. T., Teal, P. E., Malik, R. U., Edison, A. S., Sternberg, P. W., and Schroeder, F. C. (2008). A blend of small molecules regulates both mating and development in Caenorhabditis elegans. Nature 454, 1115-1118. Abstract Article 
Srinivasan, J., von Reuss, S. H., Bose, N., Zaslaver, A., Mahanti, P., Ho, M. C., O'Doherty, O. G., Edison, A. S., Sternberg, P. W., and Schroeder, F. C. (2012). A modular library of small molecule signals regulates social behaviors in Caenorhabditis elegans. PLoS Biol 10, e1001237. Abstract Article

Terrillon, S., and Bouvier, M. (2004). Roles of G-protein-coupled receptor dimerization. EMBO Rep. 5, $30-34$. Abstract Article

Thorson, J. S., Lo, S. F., Ploux, O., He, X., and Liu, H. W. (1994). Studies of the biosynthesis of 3,6-dideoxyhexoses: molecular cloning and characterization of the asc (ascarylose) region from Yersinia pseudotuberculosis serogroup VA. J. Bacteriol. 176, 5483-5493. Abstract

von Reuss, S. H., Bose, N., Srinivasan, J., Yim, J. J., Judkins, J. C., Sternberg, P. W., and Schroeder, F. C. (2012). Comparative metabolomics reveals biogenesis of ascarosides, a modular library of small molecule signals in $C$ elegans. J. Am. Chem. Soc. 134, 1817-1824. Abstract Article

Vowels, J. J., and Thomas, J. H. (1992). Genetic analysis of chemosensory control of dauer formation in Caenorhabditis elegans. Genetics 130, 105-123. Abstract

White, J. Q., and Jorgensen, E. M. (2012). Sensation in a single neuron pair represses male behavior in hermaphrodites. Neuron 75, 593-600. Abstract Article

White, J. Q., Nicholas, T. J., Gritton, J., Truong, L., Davidson, E. R., and Jorgensen, E. M. (2007). The sensory circuitry for sexual attraction in Celegans males. Curr. Biol. 17, 1847-1857. Abstract Article

Yamada, K., Hirotsu, T., Matsuki, M., Butcher, R. A., Tomioka, M., Ishihara, T., Clardy, J., Kunitomo, H., and Iino, Y. (2010). Olfactory plasticity is regulated by pheromonal signaling in Caenorhabditis elegans. Science 329, 1647-1650. Abstract Article

Zhang, S. O., Box, A. C., Xu, N., Le Men, J., Yu, J., Guo, F., Trimble, R., and Mak, H. Y. (2010). Genetic and dietary regulation of lipid droplet expansion in Caenorhabditis elegans. Proc. Natl. Acad. Sci. U. S. A. 107, 4640-4645. Abstract Article

Zwaal, R. R., Mendel, J. E., Sternberg, P. W., and Plasterk, R. H. (1997). Two neuronal G proteins are involved in chemosensation of the Caenorhabditis elegans dauer-inducing pheromone. Genetics 145, 715-727. Abstract

All WormBook content, except where otherwise noted, is licensed under a Creative SOMIERIGHISRESERVED Commons Attribution License. 